\title{
Stoffbuchhaltung für eine Müllverbrennungsanlage
}

Dr. Hasan Belevi, Madeleine Langmeier, Hermann Mönch, Yvonne Turban, Thomas Müller, Prof. Dr. Peter Baccini

\begin{abstract}
Dr. Hasan Belevi Studium Chemie Ingenieur und Promotion an der ETH Zürich. Selt 1985 wissenschaftlicher Mitarbeiter an der Eidgenössischen Anstalt für Wasserversorgung, Abwasserreinigung und Gewässerschutz (EAWAG). Leiter der Forschungsgruppe Entsorgungstechnik an der Abteilung Stoffhaus hait und Entsorgungs. technik, Lehrbeauftragter der ETH Zürich.
\end{abstract}

Madaleine Langmeier Lehre als Chemie Labo. rantin. Technische Mitar beiterin an der Eid. genössischen Anstalt für Wasserversorgung Abwasserreinigung und Gewässerschutz (EAWAG) Abteilung Stofihaushalt und Entsorgungstechnik.

Hermann Mönch Lehre als Chemie-Labo. rant. Technischer Mitar. beiter an der Eidgenös. sischen Anstalt für Was. serversorgung, Abwas. serreinigung und $\mathrm{Ge}$ wässerschutz (EAWAG) Abteilung Stoffhaushalt und Entsorgungstechnik.

Yvonne Turban Lehre als Drogistin. Chemie-Laborantin an der Müllverbrennungs anlage Oftringeri.

\section{Thomas Müller} Studium der Cheme. Geschaftsleiter der Müllverbrennungsanlage Oftringen.

\section{Prof. Dr.}

Peter Baccini

Studium der Chemie

und Promotion an der ETH Zurich, Ordenti. cher Prolessor fur Stoff. haushalt und Entsor gungstechnik an der ETH Zurich.Lerter der Abtellung Stoffhaushalt und Entsorgungstechnik an der Eidgenossischen Anstalt fur Wasserver. sorgung, Abwasserrein. gung und Gewasserschutz (EAWAG).

\section{Übersicht}

Eine Methode für die kontinuierliche Erfassung, Visualisierung und Interpretation der Güter- und Stoffflüsse in einer Müllverbrennungsanlage wird vorgestellt. Sie dient als Grundlage sowohl für ein betriebliches Umweltmanagement als auch für ein marktorientiertes Ressourcenmanagement. Sie erlaubt zudem eine permanente Qualitätskontrolle und Qualitätssicherung. Die ersten Ergebnisse zeigen, daß die Methode eine Erfassung der Güterflüsse mit einer zeitlichen Auflösung von einigen Tagen ermöglicht. Die Stoffflüsse können für Zeitabschnitte von einigen Wochen bestimmt werden. Eine bessere Auflösung ist grundsätzlich möglich, erfordert jedoch einen höheren finanziellen Aufwand

\section{Einführung}

Müllverbrennungsanlagen (MVA) können in einer Volkswirtschaft folgende Funktionen erfüllen:

1. Konzentrierung diffus verteilter Güter heterogener Zusammensetzung an einem Ort (von einigen Hunderttausend Personen, auf rund $10^{5}$ Privathaushalten und $10^{4}$ Unternehmungen verteilt, auf einer Fläche von $10^{2}-10^{3} \mathrm{~km}^{2}$ ).

2. Chemische Umwandlung potentiell schädlicher Gütergemische in umweltverträgliche Produkte.

3. Raffinierung der Produkte in wiederverwertbare Güter wie Energieträger, Baumaterialien, Erze.

4. Indikatoren für stoffliche Veränderungen im Einzugsgebiet.

Funktionen 1 und 2 werden heute als selbstverständlich erachtet. Sie entsprechen einerseits der langen Tradition der Städtereinigung, welche zur Aufgabe der öffentlichen Hand gehört und andererseits den jüngeren Ansprüchen des Umweltschutzes. Die dritte Funktion wird im Falle der Energie in den meisten Fällen erfüllt. Hingegen steht eine Realisierung der Verwertung fester Rückstände erst in den Anfängen. Frühere Untersuchungen haben bereits aufgezeigt $[1,2]$, daß Siedlungsabfälle für einige Stoffe (wie z. B. Kupfer, Zink, Cadmium) wichtige Förderbänder im Gesamtumsatz einer Volkswirtschaft darstellen. Es handelt sich meist um nicht erneuerbare Stoffe, die ubiquitär eingesetzt werden. Mittel- und langfristig betrachtet bilden ihre anthropogenen Lager schon heute eine Ressource, die in ihrer Grösse jenen der heute global verfügbaren geogenen Lagerstätten gleichkommt [3]. Auch gesteinsähnliche Materialien wie MVA-Schlacke sind mengenmässig (100-200 kg pro Einwohner und Jahr) gewichtig!, liegen sie doch in der gleichen Größenordnung wie z. B. die jährlich benö-

1 Dies trifft zu für eine Region, welche samtliche Siedlungsabfälle in einer MVA behandelt. tigte Zementmenge. MVA sind großtechnische Raffinierungsanlagen mit dem Potential, Gesteine und Erze herzustellen.

Die vierte Funktion basiert auf einer Perspektive, welche bis heute kaum wahrgenommen wird. Müllverbrennungsanlagen und Abwasserreinigungsanlagen sind nicht nur Orte, wo Konzentrate des urbanen Stoffwechsels gebildet werden. Sie sind auch grosstechnische Aufschlussapparaturen, in deren Produkten das stoffliche Profil eines Siedlungsgebietes wie „Fingerabdrücke" gespeichert wird (Abbildung 1.1). Am Beispiel von Abwasserreinigungsanlagen konnte dies kürzlich im Klärschlamm differenziert aufgezeigt werden [4]. Im Klärschlamm lassen sich stoffliche Veränderungen im Einzugsgebiet der ARA erkennen und deren Ursachen zuordnen. In Müllverbrennungsanlagen wurde dieses Potential bis heute nur in Einzelfällen und sporadisch genutzt. Mittelfristig sprechen zwei Gründe für den Einbezug der Funktionen 3 und 4 ins MVAPf]ichtenheft:

a) Die aus Umweltschutzgründen stark ausgebaute und damit teure Behandlungstechnik braucht relativ wenig Zusatzaufwand (ökonomisch betrachtet), um die Produktequalität der festen Rückstände wesentlich zu verbessern. Auch die Qualitätsanforderung für die Deponierung fester Rückstände wird zunehmen, weil ein Export in dünn besiedelte Gebiete langfristig kaum mehr möglich ist und die künftigen Deponien „mitten in der Stadt" sein werden [5].

b) Der Schritt von der „Reinigungstechnik“ zu einem Stoffstrommanagement [6] zwingt die MVA früher oder später zu dieser Funktion. Eine kluge Nutzung der stofflichen Informationen der MVA über ihr Einzugsgebiet wird breit angelegten Monitorprogrammen aus ökonomischen Gründen weit überlegen sein. Zudem werden auch MVA-Betreiber dazu angehalten werden, sich dem Standard eines Umweltaudit-Systems anzupassen, wie es die Wirtschaft in ihren Unternehmungen seit einigen Jahren konsequent entwickelt.

Für die Funktionen 1 und 2 ist es ausreichend, mit einer einfachen Massenbilanz der Güter und einer Kontrolle der Emissionen (Einhaltung der Emissionsgrenzwerte) zu arbeiten. Dafür genügen die schon heute verfügbaren betriebswirtschaftlichen und analytischen Methoden. Will man dagegen auch die Funktionen? Ind 4 erfüllen, so braucht es eine betriebliche Energie- und Stoffbuchhaltung, hier abgekürzt als Stoffbuchhaltung tituliert.

Die Stoffbuchhaltung basiert auf der Methode der Stoffflussanalyse [3,7]. Ein Unternehmen wird als Stoffhaushaltssytem begriffen, für das die relevanten Prózesse, Güter und Stoffe erfasst werden. Die dazu notwendige Datenbank ist eine Erweiterung des betrieblichen Rechnungswesen. Diese erweiterte Buchhaltung erlaubt prinzipiell eine kontinuierliche Erfassung, Visualisierung und Interpretation des betrieblichen Energie- und Stoffhaushaltes. Sie dient zudem als Grundlage sowohl für ein betriebliches Umweltmanagement als auch für ein marktorientiertes Ressourcenmanagement [8].

Für MVA existieren noch keine Stoffbuchhaltungen 
der obengenannten Art. Seit Mitte der achtziger Jahre $[9,10]$ wurden Stoffflußanalysen an MVA durchgeführt. Es handelte sich aber um Momentaufnahmen. Solche Meßkampagnen sind allerdings sehr aufwendig und damit teuer. Die Ergebnisse aus diesen Arbeiten erlauben aber, erste maßgeschneiderte Meßprogramme zu entwickeln, welche für eine MVA-Stoffbuchhaltung eingesetzt werden können. Zudem bieten sie dem Betreiber zusätzliche verfahrenstechnische Informationen, die er für eine permanente Qualitätskontrolle und -sicherung einsetzen kann, Die hier vorgestellte Arbeit zeigt den Aufbau eines Messprogrammes für die Alimentierung einer solchen Stoffbuchhaltung. Insbesondere sollen folgende Fragen (exemplifiziert an einer konkreten Anlage) beantwortet werden:

1. Wie wird das System für eine MVA-Stoffbuch haltung aufgebaut?

2. Was wird wie beprobt und gemessen (physikalische und chemische Analysen)?

3. Wie wird die Stoffbuchhaltung erstellt und angewendet?

\section{Methode}

\subsection{Konzept}

Die Stoffflußanalyse ist ein naturwissenschaftliches Verfahren, um für einen definierten Raum in einer' bestimmten Zeitperiode den Stoffumsatz zu quántifizieren. Sie wurde bis heute für unterschiedliche Systeme wie technische Verfahren, landwirtschaftliche Böden, Privathaushalte und ganze Regionen angewandt $[1,3]$.

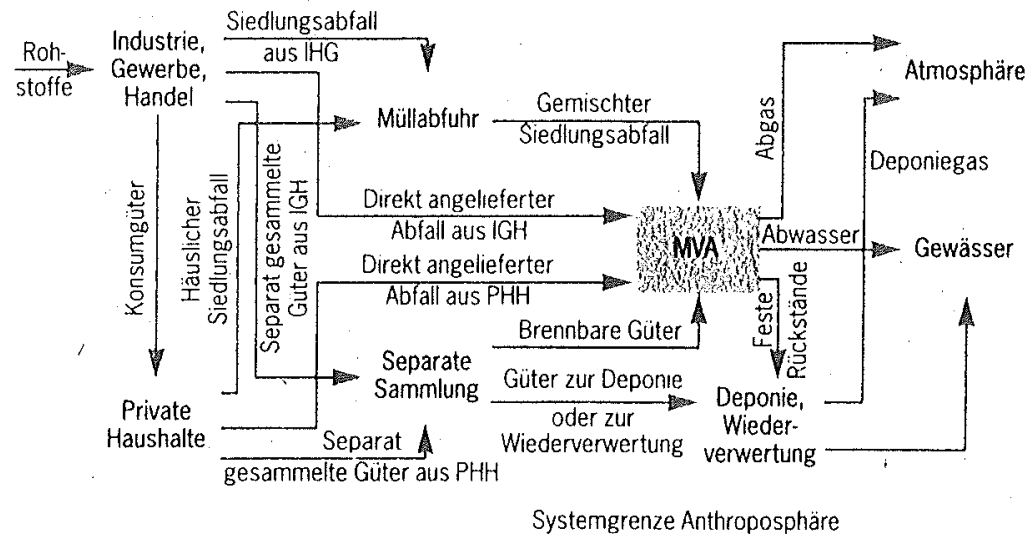

Abbildung 1.1: Müllverbrennungs anlage als techni-

Die betriebliche Stoffbuchhaltung auf der Basis der Stoffflußanalysen verlangt folgende Schritte [8]:

Darstellung des Betriebes als Stoffhaushaltsystem

(Systemanalyse), in welchem die relevanten Prozesse und Güter miteinander verknüpft und mit Her-

kunfts-und Zielprozessen außerhalb des Betriebes verbunden werden.

Der zweite Schritt führt zur Messung(Datenerfassung) der Güterflüsse und der Stoffkonzentrationen der Güter. Eine Sichtung der bereits vorhandenen Daten des Betriebes, die aus verschiedenen Quellen stammen (z. B. betriebliches Rechnungswesen, Prozeßkontrolle, Emissionskontrolle), identifiziert die vorhandenen Datenlücken, welche gemessen werden müssen. Bei der Datenerfassung spielt der scher Prozess inner halb der Anthroposphäre
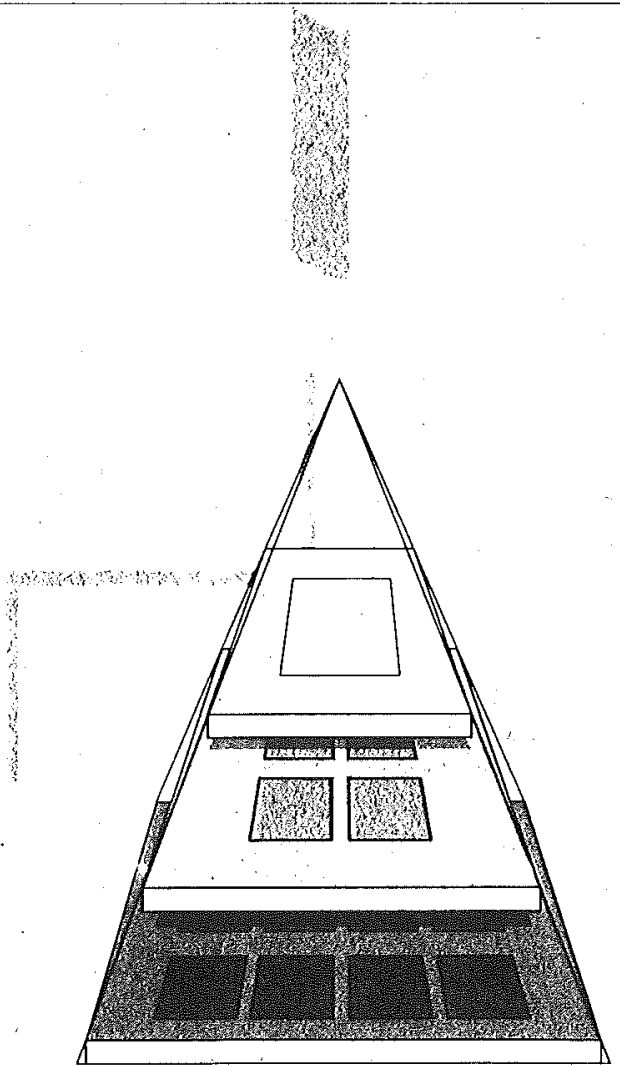

Informieren Sie sich unter http://www.ifu.com/umberto

\section{Software für den Umweltschutz}

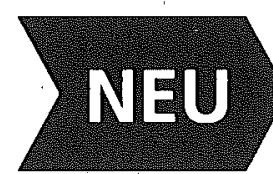

umberto Version 3.0

Die Software für Stoff- und Energiestromanalysen, Produktökobilanzen und betriebliche Umweltbilanzen

\section{Jetzt:}

- Übersichtliche hierarchische Netzebenen

- Integrierte (Umwelt)Kostenrechnung

- Anschauliche Sankey-Darstellung

- Vielseitige Material- und Stoff-Datenbank

- Fortschrittliche 32-bit-Client-Server-Anwendung

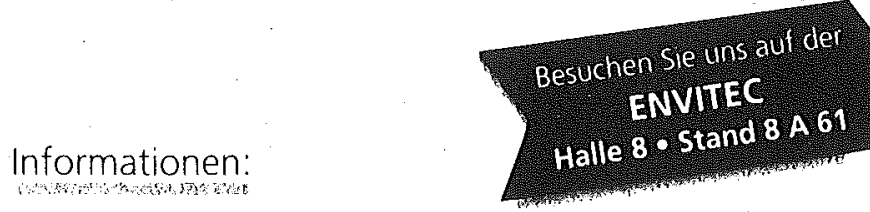

Hamburg: Tel. (040) 4800 09-0, Fax (040) 4800 09-22 
Abbildung 2.2.1: Systemanalyse der MVA-Oftringen

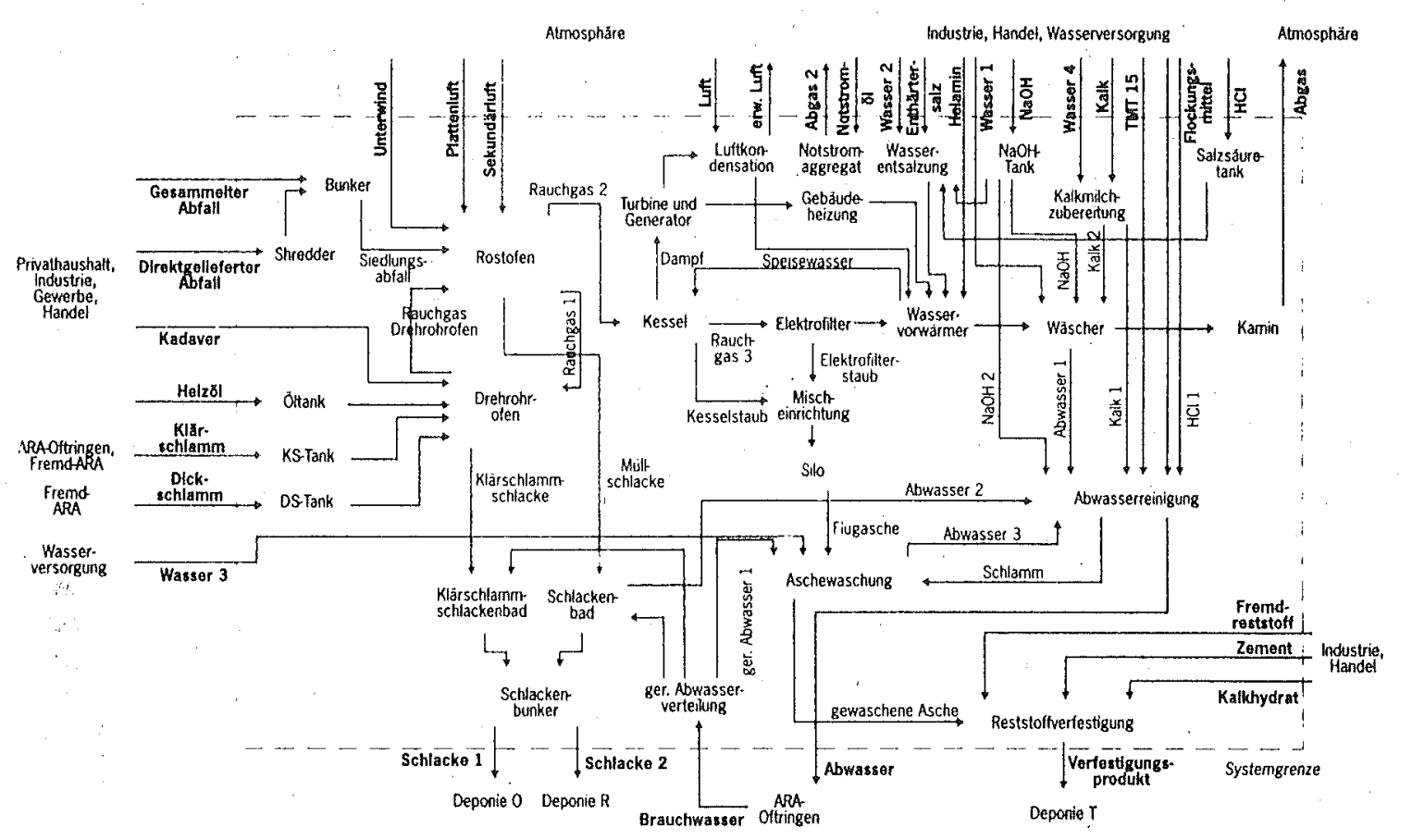

ökonomische Aufwand eine Schlüsselrolle. Die Auswahl der Indikatorstoffe erfolgt nach verschiedenen Kriterien. Einmal ist es notwendig, jene Stoffe zu wählen, welche die wichtigsten Güter am besten charakterisieren. Im weiteren sind Stoffe zu berücksichtigen, die gemäß Erfahrung die Umweltkompartimente stark belasten können. Schließlich kann es aus methodischen Gründen nützlich sein, Stoffe auszuwählen, welche interne Prozeßveränderungen am besten quantifizieren. Nach erfolgter Ergänzung der Daten ist es möglich, eine Stoffbilanz zu erstellen und die erste Modifizierung des Systems vorzunehmen.

- Nach der Modifizierung des Systems werden die weiter zu erfassenden Größen und die Erfassungsperioden festgelegt, um eine kontinuierliche Stoffbuchhaltung durchführen zu können. Je nach erhaltenen Resultaten sowie Kosten und Nutzen können am kontinuierlichen Datenerfassungsprogramm weitere Veränderungen vorgenommen werden.

\subsection{Systemanalyse}

Die Entwicklung der Stoffbuchhaltung erfolgte in $\mathrm{Zu}$ sammenarbeit mit der Müllverbrennungsanlage in Oftringen, Kanton Aargau, Schweiz. Über 160.000 Einwohner aus Verbandsgemeinden und Zuliefergemeinden der Kantone Aargau und Luzern sind der MVA Oftringen angeschlossen. Siedlungsabfälle werden sowohl von städtischen als auch von ländlichen Gebieten gesammelt. Direktanlieferungen von haushaltsabfallähnlichen Gütern aus Industrie, Gewerbe und Handel machen im Verbandsgebiet bis zu $50 \%$ der verbrannten Abfälle aus. Die direkt angelieferten Abfälle werden zuerst in einer Rotationssperrgutschere zerkleinert, bevor sie in den Bunker gelangen. Die Anlage verbrennt Müll in einem Rostofen mit einem Vorschubrost und einer maximalen Kapazität von $10.5 \mathrm{t} / \mathrm{h}$.
Parallel zur Siedlungsabfallverbrennung werden Klärschlamm der ARA-Oftringen (auf demselben Areal), Frischschlamm und ausgefaulter Schlamm (Dickschlamm) anderer Kläranlagen in einem Drehrohrofen mit einer maximalen Kapazität von $0.6 \mathrm{t}$ Trockensubstanz pro Stunde verbrannt. Die Rohgase werden durch einen Elektrofilter und einen 3-stufigen Rauchgaswäscher gereinigt. Die Anlage enthält eine Abwasseraufbereitungsanlage und eine Reststoffverfestigungsanlage. Die Lieferanten der Anlageteile:

- Feuerung, Kessel und Economizer, Drehrohrofen, Elektrofilter: NOELL-Abfalltechnik, Schlieren/CH mit Unterlieferanten Wamser, Neuss/D, Pleq, Köln/D, Elex, Zürich/CH

Rauchgaswäscher, Abwasseraufbereitungsanlage: Techfina, Winterthur/CH mit Unterlieferant LAB, Lyon/F

- Stromerzeugung: $\mathrm{ABB}, \mathrm{Baden} / \mathrm{CH}$

Abbildung 2.2.1 zeigt die Systemanalyse der Anlage. Hier werden einfachheitshalber die gleichen Begriffe eingesetzt wie sie in der Anlage verwendet werden. Die Anlage verbrennt Müll im Rostofen und Klärschlamm im Drehrohrofen. Die Müllschlacke und die Klärschlammschlacke werden getrennt in Wasserbädern abgekühlt und gemeinsam im Schlackenbunker zwischengelagert, bevor sie zu den Deponien transportiert werden.

Ein Teil des Rohgases aus dem Rostofen wird in den Drehrohrofen abgeleitet zur Trocknung des Klärschlammes und auch als Sauerstoffquelle für die Verbrennung des Klärschlammes. Die Gase aus dem Drehrohrofen gelangen wieder in den Rostofen.

Nach der Abkühlung im Kessel wird das Rauchgas in einem Elektrofilter und einem dreistufigen Wäscher gereinigt und gelangt über den Kamin in die Atmosphäre. Im Kessel wird mit der Energie des Rauchgases überhitzter Dampf erzeugt, welcher sich in einer 
Dampfturbine zur Stromerzeugung entspannt. Für das Dampferzeugungssystem und die Gasreinigung werden verschiedene Hilfsstoffe benötigt.

Der Staub aus dem Kessel und dem Elektrofilter wird gemischt und im Silo zwischengelagert, bevor er mit dem Schlamm aus der Abwasserreinigung in der Aschewaschung gereinigt wird. Die gewaschene Asche wird mit angelieferten Fremdreststoffen anderer Anlagen in der Halle der Reststoffverfestigung gelagert. Wenn sich genug Asche angesammelt hat, werden sie und die Fremdreststoffe mit Kalkydrat und Zement vermischt und in noch feuchtem Zustand zur Deponie transportiert, wo sich die Masse verfestigt.

Das gesamte in der Anlage anfallende Abwasser (aus Wäscher, Klärschlamm- und Müllschlackenbad, Aschewaschung) wird in der Abwasserreinigung aufbereitet und in die ARA-Oftringen geleitet. Im Schlackenbad, Klärschlammschlackenbad und in der Aschewaschung wird das gereinigte Wasser aus der ARA-Oftringen als Brauchwasser eingesetzt.

Die Verbrennung, Rauchigasreinigung und Abwasserreinigung sind kontinuierlich in Betrieb während 24 Stunden. Die Aschewaschung ist diskontinuierlich an zwei bis drei Tagen pro Woche im Einsatz. Die Reststoffverfestigung wird nur an ein bis zwei Tagen pro zwei bis vier Wochen betrieben, wenn genügend Asche angefallen ist

\subsection{Bèstimmung der Güterflüsse}

In einer Vorbereitungsphase wurden Stoffflußanalysen von Subsystemen durchgeführt. Durch diese Analysen wurden auch die internen Flüsse im System abgeschätzt. Die Güterflüsse zwischen einigen Prozessen konnten nur durch sehr hohen Aufwand abgeschätzt werden. Dadurch schien eine Bestimmung der internen Güter- und Stof'́flüsse in einer kurzen Zeitperiode (z.B. 2 bis 4 Wochen) mit den in einer Müllverbrennungsanlage zur Verfügung stehenden Mitteln nicht vernünftig. /uus diesem Grund wurden nuralle Input- und Outpucflüsse, die in und aus dem System geführt werden, für die Stoffbuchhaltung erfaßt. Die inneren Flüsse werden mit einer besonderen Stoffflußanalyse einmalig bestimmt. Diese Stoffflußanalyse soll im Rahmen einer späteren Publikation detailliert beschrieben werden [17].

In der MVA Oftringen wird ein sehr grosser Teil der Input- und Outputgüter für die Fakturierung, für die Zusammenstellung des Jahresberichtes oder in den Betriebsprotokollen regelmäßig erfaßt. Auch existieren einige Angaben als Papierausdruck aus dem Prozeßleitsystem. Für die Stoffbuchhaltung müssen deshalb keine zusätzlichen Messungen durchgeführt werden. Tabelle 2.3.1 gibt einen Überblick über die Erfassung der Input- und Outputgüter.

\subsection{Bestimmung der Stoffkonzentrationen}

\subsubsection{Auswahl der Stoffe}

Folgende Indikatorstoffe wurden gewählt:

- Natrium, Magnesium, Aluminium, Silizium, Kalium und Calcium (Metalle und Metalloide):

Diese Elemente bilden die Hauptelemente in den festen Rückständen der Müllverbrennungsanlage

\begin{tabular}{llll}
\hline Gut & Messort & Zeitpunkt & $\begin{array}{l}\text { Erfassung für die } \\
\text { Erfassung }\end{array}$ \\
& Stoffbuchhaltung
\end{tabular}

\begin{tabular}{|c|c|c|c|}
\hline INPUT & & & \\
\hline Gesammelter Abfall & Brückenwaage & bei Einfahrt & täglich \\
\hline Direkt gelieferter Abfall & Brückenwaage & bei Einfahrt & täglich \\
\hline Kadaver & Brückenwaage & bei Einfahrt & täglich \\
\hline Klärschlamm & Waage am Förderband. & kontinuierlich. & täglich \\
\hline Dickschlamm & Brückenwaage & bei Einfahrt & täglich \\
\hline Fremdreststoff & Brückenwaage & bei Lieferung & bei Lieferung \\
\hline Wasser $1-5$ & Zentrale Wasseruhr & kontinuierlich & täglich \\
\hline Brauchwasser & Wasseruhr & bei Lieferung & bei Lieferung \\
\hline $\begin{array}{l}\text { Heizöl, Salzsäure (HCi), } \\
\text { Natron-Lauge (NaOH), Kalk, } \\
\text { TMT 15, Flockungshilfs- } \\
\text { mittel (FHM), Kalkhydrat, } \\
\text { Zement, Enthärtersalz, } \\
\text { Helamin }\end{array}$ & Brückenwaage & bei Lieferung & bei Lieferung \\
\hline $\begin{array}{l}\text { Unterwind, Plattenluft, } \\
\text { Sekundärluft }\end{array}$ & Prozessleitsystem & kontinuierlich & $\begin{array}{l}\text { täglich ein } \\
\text { Momentanwert }\end{array}$ \\
\hline
\end{tabular}

\section{OUTPUT}

\begin{tabular}{llll} 
Schlacke & Brückenwaage & bei Ausfahrt & täglich \\
\hline Verfestigungsprodukt & Brückenwaage & bei Ausfahrt & $\begin{array}{l}\text { täglich während } \\
\text { der Verfestigung }\end{array}$ \\
\hline Abwasser & Wasseruhr & $\begin{array}{l}\text { kontinuierlich } \\
\text { kumulativ }\end{array}$ & täglich \\
\hline Abgas & Prozessleitung & kontinuierlich & $\begin{array}{l}\text { täglich ein } \\
\text { Momentanwert }\end{array}$ \\
\hline
\end{tabular}

und beeinflussen die Wiederverwertbarkeit oder

das Langzeitverhalten in Deponien.

Kohlenstoff, Phosphor, Schwefel und Chlor (Nichtmetalle) :

Kohleristoff ist eines der wichtigsten Hauptelemente im Müll. Die wichtigste chemische Umwandlung im Ofen ist die Oxidation des Kohlenstoffes zu $\mathrm{CO}_{2}$. Diese Reaktion soll möglichst vollständig ablaufen, um eine gute Qualität der Produkte (Abgas, Schlacke, Rückstände der Rauchgasreinigung) zu gewährleisten.

Phosphor ist im Klärschlamm ·grossen Konzentrationen vorhanden ( 8 bis $45 \mathrm{~g} / \mathrm{kg}$ Trockensubstanz) und gelangt fast vollständig in die Schlacke.

Bei der Verbrennung des Schwefels entsteht SO2 und muss vom Rauchgas entfernt werden. Die gasseitigen Emissionen sind durch Verordnungen begrenzt [12].

Bei der Verbrennung von chlorhaltigen Verbindungen entsteht Salzsäure welche vom Rauchgas entfernt werden muss. Gasseitige Emissionen sind durch Verordnungen begrenzt [12]. Die leichtlöslichen Chloridsalze können in Deponien aus den Verbrennungsrückständen herausgewaschen werden [18].

Kupfer, Zink, Cadmium und Blei (Schwermetalle): 


\begin{tabular}{|c|c|c|c|}
\hline \multirow[t]{2}{*}{ Gut } & \multirow[t]{2}{*}{ Probenahme } & \multicolumn{2}{|c|}{ Chemische Analysen } \\
\hline & & Einzelprobe & Mischprobe ${ }^{11}$ \\
\hline \multicolumn{4}{|l|}{ INPUT } \\
\hline $\begin{array}{l}\text { Gesammelter Abfall, } \\
\text { Direkt gelieferter Abfall, } \\
\text { Kadaver }\end{array}$ & keine & keine & keine \\
\hline Klärschlamm & $\begin{array}{l}\text { Eine Probe pro Woche an } \\
\text { alternierenden Tagen }\end{array}$ & Zink und Kupfer ${ }^{21}$ & $\begin{array}{l}\text { Alle Stoffbuch- } \\
\text { haltungselemente }\end{array}$ \\
\hline Dickschlamm & $\begin{array}{l}\text { Eine Probe pro Woche an } \\
\text { alternierenden Tagen }\end{array}$ & Zink und Kupfer & $\begin{array}{l}\text { Alle Stoffbuch- } \\
\text { haltungselemente }\end{array}$ \\
\hline Fremdreststoff & $\begin{array}{l}\text { Eine Probe von jeder } \\
\text { Sorte bei der Lieferung }\end{array}$ & keine & $\begin{array}{l}\text { Alle Stoffbuch- } \\
\text { haltungselemente }\end{array}$ \\
\hline Wasser $1-5$ & keine & keine & keine \\
\hline $\begin{array}{l}\text { Brauchwasser; Natron- } \\
\text { lauge, Salzsäure, } \\
\text { gebrannter Kalk, } \\
\text { Kalkhydrat, Zement }\end{array}$ & $\begin{array}{l}\text { Eine Probe während der } \\
\text { Vorbereitungsphase }\end{array}$ & $\begin{array}{l}\text { Alle Stoffbuch- } \\
\text { haltungselemente }\end{array}$ & keine \\
\hline $\begin{array}{l}\text { Helamin, Enthärtersalz, } \\
\text { FHM, TMT 15, Heizöl }\end{array}$ & keine & keine & keine \\
\hline $\begin{array}{l}\text { Unterwind, Plattenluft, } \\
\text { Sekundärluft }\end{array}$ & keine & keine & keine \\
\hline
\end{tabular}

\begin{tabular}{llll}
$\begin{array}{l}\text { OUTPUT } \\
\text { Schlacke }\end{array}$ & $\begin{array}{l}\text { Eine Probe pro Woche an Zink und Kupfer } \\
\text { alternierenden Tagen }\end{array}$ & $\begin{array}{l}\text { Alle Stoffbuch- } \\
\text { haltungselemente }\end{array}$ \\
\hline Verfestigungsprodukt & $\begin{array}{l}\text { Eine Probe bei jeder } \\
\text { Verfestigung }\end{array}$ & Zink und Kupfer & $\begin{array}{l}\text { Alle Stoffbuch- } \\
\text { haltungselemente }\end{array}$ \\
\hline Abwasser & $\begin{array}{llll}\text { Eine Probe pro Woche an Zink und Kupfer } \\
\text { alternierenden Tagen }\end{array}$ & $\begin{array}{l}\text { Alle Stoffbuch- } \\
\text { haltungselemente }\end{array}$ \\
\hline Abgas & $\begin{array}{l}\text { Einmal im Jahr nach } \\
\text { BUWAHL-Richtlinien [16] }\end{array}$ & $\begin{array}{l}\text { Nach BUWAL- } \\
\text { Richtlinien [16] }\end{array}$ & $\begin{array}{l}\text { Nach BUWAL- } \\
\text { Richtlinien [16] }\end{array}$ \\
\hline
\end{tabular}

"Drei-Monate-Mischprobe

2) Nur wenn Information über Herkunfts- und Zielprozesse erwünscht wird (vgl. Kap. 4)

"Mengenproportionale Mischung jeder Sorte

Tabelle 2.4.1:

Bestimmung der Stoffkonzentrationen
Die Emissionen sind durch Verordnungen begrenzt. Kupfer wird hauptsächlich in die Schlacke und Cadmium hauptsächlich in die Rückstände der Rauchgasreinigung transferiert. Deren Flüsse liefern erste Hinweise über den Input im Siedlungsabfall. Zink und Blei werden jedoch sowohl in die Schlacke als auch in die Rückstände der Rauchgasreinigung transferiert. Diese Transfers werden stark auch durch die Prozessbedingungen in der Anlage beeinflußt. Die Verteilung von Zink und Blei kann folglich auch Hinweise über den Prozeßverlauf liefern.

\subsubsection{Meßprogramm}

Tabelle 2.4.1 zeigt die Methode zur Bestimmung der Stoffkonzentrationen. Der gesammelte und direktgelieferte Abfall sowie die Kadaver werden nicht beprobt. Die durchschnittlichen Konzentrationen im gesamten Abfallinput (gesammelter und direkt angelieferter Abfall und Kadaver) werden durch Stoffbilanzen über die Anlage berechnet. Der Güterfluß der Kadaver am gesamten Input beträgt weniger als $1 \%$ und kann deshalb die mittlere Zusammensetzung der eingeführten Siedlungsabfälle nicht beeinflussen.
Trinkwasser, Helamin, Enthärtersalz, FHM, TMT15 und Heizöl werden nicht beprobt. Die Konzentrationen werden anhand von Herstellerangaben oder Literaturwerten abgeschätzt. Die Beiträge an die Stoffflüsse dieser Güter für alle Elemente sind vernachlässigbar klein. Der Inputfluß der zu untersuchenden Elemente durch Luft wird auch vernachlässigt.

Klärschlamm, Dickschlamm, Schlacke und Abwasser werden einmal pro Woche beprobt und das Verfestigungsprodukt bei jeder Verfestigung (ca. alle 2 bis 4 Wochen). Alle drei Monate werden Mischproben von diesen Gütern hergestellt und die Konzentrationen der Elemente, die in Abschnitt 2.4.1 angegeben worden sind (Stoffbuchhaltungselemente), gemessen. Die Konzentrationen von Zink und Kupfer werden zusätzlich von allen Einzelproben bestimmt. Die Gründe für die Wahl von Zink und Kupfer werden in Abschnitt 3.2 angegeben.

Von jeder Sorte der Fremdreststoffe wird ca. alle 3 Monate eine Mischprobe hergestellt und nach allen Stoffbuchhaltungselementen analysiert. Brauchwasser, Natronlauge, Salzsäure, gebrannter Kalk, Kalkhydrat und Zement werden einmal beprobt und nach allen Stoffbuchhaltungselementen analysiert. Wenn neue Qualitäten für Betriebsmittel (z. B. für Zement) eingesetzt werden, werden sie analysiert. Die Abgase werden mit regelmäßigen Emissionsmessungen durch externe Firmen in Erfüllung der Luftreinhalteverordnung überprüft' [12]. Die Konzentrationen werden anhand dieser Daten erfaßt.

Dieser Plan für die Bestimmung der Stoffkonzentrationen stellt bezüglich der Probenanzahl eine maximale Variante dar. Je nach erhaltenen Resultaten kann die Probenahme in größeren Abständen durchgeführt werden. Die Probenahme der Schlacke kann zum Beispiel alle zwei Wochen durchgeführt werden, da der Aufwand für die Schlackeprobenahme und Aufbereitung sehr hoch ist (vgl. Abschnitt 2.4.3). Das Verfestigungsprodukt sollte jedoch bei jeder Verfestigung beprobt werden, da dadurch sehr viel Information gewonnen wird. Auf die Analysen der einzelnen $\mathrm{Ab}$ wasser- und Schlammproben kann verzichtet werden. Die Stoffflußanalysen, die in der Vorbereitungsphase durchgeführt wurden [17], haben gezeigt, dass für die hier gewählten Indikatorelemente die Beiträge dieser Güter in den Stoffflüssen der MVA sehr klein sind (Phosphor ist eine Ausnahme). Die Analysen der Mischproben sollten jedoch weitergeführt werden, da sie auch Informationen über Herkunftsprozesse liefern (ARA Oftringen und Fremd-Abwasserreinigungsanlagen), die für das MVA Marıagement wichtig sein können. In der vorliegenden Arbeit wurden jedoch in den ersten 4 Monaten der kontinuierlichen Stoffbuchhaltung alle Abwasser" und Schlammproben nach Zink und Kupfer analysiert, um die obigen Aussagen überprüfen zu können.

\subsubsection{Probenahme und Probenaufbereitung}

\subsubsection{Klärschlamm und Dickschlamm}

Vom Klärschlamm und Dickschlamm werden alle 15 Minuten während einer Stunde Proben genommen (Total je 5 Proben) und vermischt. Anschließend wird eine Teilmenge von ca. $3 \mathrm{~kg}$ in einen Plastiksack ge- 
geben. Die Proben werden bei $110^{\circ} \mathrm{C}$ getrocknet und anschließend gemahlen.

\subsubsection{Fremdreststoffe}

Mit Handschaufeln werden an verschiedenen Stellen, wo Fremdreststoffe zwischengelagert werden, 5 Proben genommen und vermischt. Anschließend wird eine Teilmenge von ca. $1 \mathrm{~kg}$ in einen Plastiksack gegeben. Die Proben werden bei $110^{\circ}$ getrocknet und anschließend gemahlen.

2.4.3.3 Brauchwasser, Zement, Kalkydrat, Natronlauge, Salzsäure, gebrannter Kalk

Die Einzelproben werden an jenen Orten genommen, wo sie zwischengelagert werden. Brauchwasser wird mit konzentrierter Salpetersäure angesäuert und in einem Kühlschrank aufbewahrt. Zement, Kalkydrat, Natronlauge, Salzsäure und gebrannter Kalk brauchen keine weitere Probenaufbereitung.

\subsubsection{Schlacke}

Die Schlackeprobenahme wird vor dem Abtransport durchgeführt. Zu dieser Zeit befindet sich ein Tagesanfall (40 bis 50 Tonnen) oder die übers Wochenende produzierte Menge (100 bis 150 Tonnen) im Bunker. Die gemischte Schlacke (Müllschlacke vermischt mit Klärschlammschlacke) wird im Bunker mit dem Kran mög. lichst homogen vermischt. Von einer Greiferladung des Mischgutes wird ca. $20 \mathrm{~kg}$ Probe mit Hilfe von Handschaufeln genommen. Das entnommene Mate- 'rial wird wieder vermischt und davon ca. $5 \mathrm{~kg}$ in einen Plastiksack gegeben.

Die Proben werden bei $110^{\circ} \mathrm{C}$ getrocknet, grobe Metallteile $>5 \mathrm{~cm}$ ) von Hand herausgenommen (Grobgut), gemahlen und durch ein Sieb mit $0.5 \mathrm{~mm} \mathrm{Ma-}$ schenweite gesiebt. Die groben Metallteile und der Siebrückstand werden gewogen, aber nicht analysiert. Für die Konzentrationen in diesen Rückständen werden Werte angenommen, die in einer anderen Studie bestimmt worden sind [17]. Die Feinfiaktion (ca. 85 bis 95 Gewichtsprozent) wird chemisch analysiert. Die Konzentrationen werden unter Berücksichtigung der Elementanteile im Grobgut und im Siebrückstand korrigiert

In der Vorbereitungsphase wurden außerdem 7 Proben an verschiedenen Stellen im durchmischten Bunker entnommen, um die Konzentrationen in diesen Proben mit den Konzentrationen in der Mischprobe zu vergleichen.

\subsubsection{Verfestigungsprodukt}

Alle 2 bis 4 Wochen werden Rückstände der Rauchgasreinigung und Abwasserbehandlung sowie Fremdreststoffe mit Zement und Kalkhydrat vermischt und in Mulden abgefüllt. Bei jeder Verfestigung fallen ca. 150 bis 250 Tonnen Verfestigungsprodukt an. Der Inhait der Mulden wird mit Handschaufeln beprobt, wobei die Proben gleichmässig über die Mulde verteilt entnommen werden. Die genommenen Proben der verschiedenen Mulden werden vermischt und davon eine

\section{LINDEMANN-ZERKLEINERUNGSTECHNIK: UMFASSEND, BEWÄHRT, FÜHREND.}

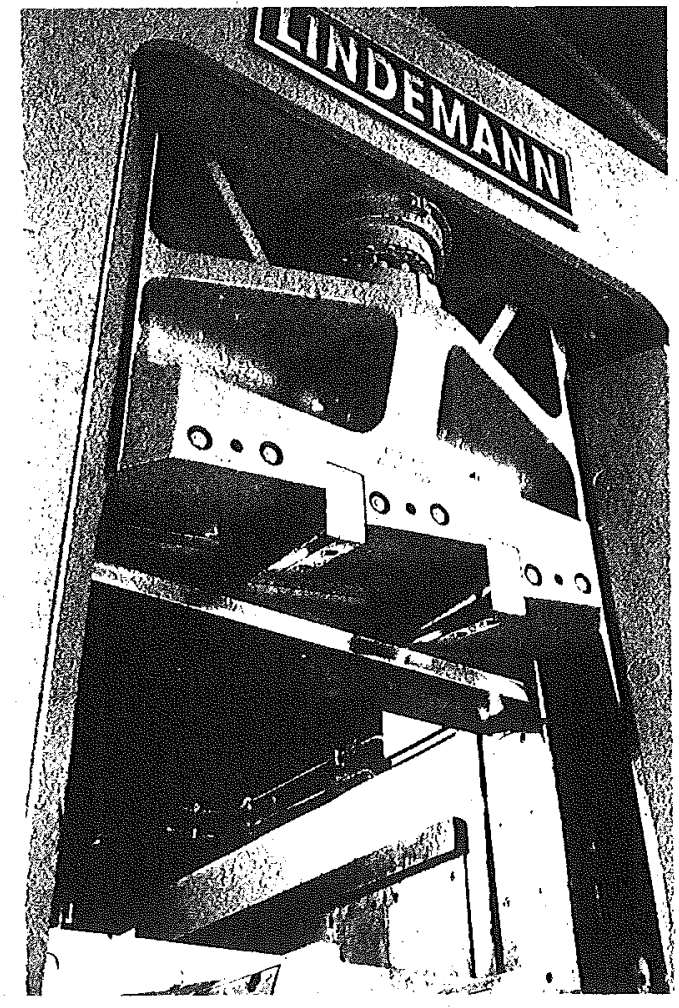

Hydrathische Sperryusschere'n
Bei der wirtschaftlichen Aufbereituny und Entsorgung von Alt- und Reststoffen zählt vor allem eine ausgereifte Technik für die zuverlässige Vorzerkleinerung.

Unsere Produkte haben sich in thermischen Abfall- und Sondermull-Aufbereitungsanlagen sowie in mechanischen und biologischen Abrallbehandlungsanlaren bewalut. Industrieunternelamen und AltstoffAufbereitungsberricbe zählen ebenso zu unseren Kunden wie Kommunen.

Zahblen Sie deshatb aut unsere jahrzehntelange Erfahrung in der Herstellung von Zerkleinerungsmaschinen und anlagen.

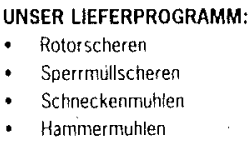

UNSER LIEFERPROGRAMM

- Rotorscheren

- Sperrmüllscheren

- Schneckenriuhler

- Hammermuhlen

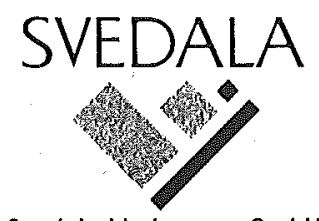

Svedala Lindemann GmbH

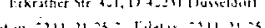

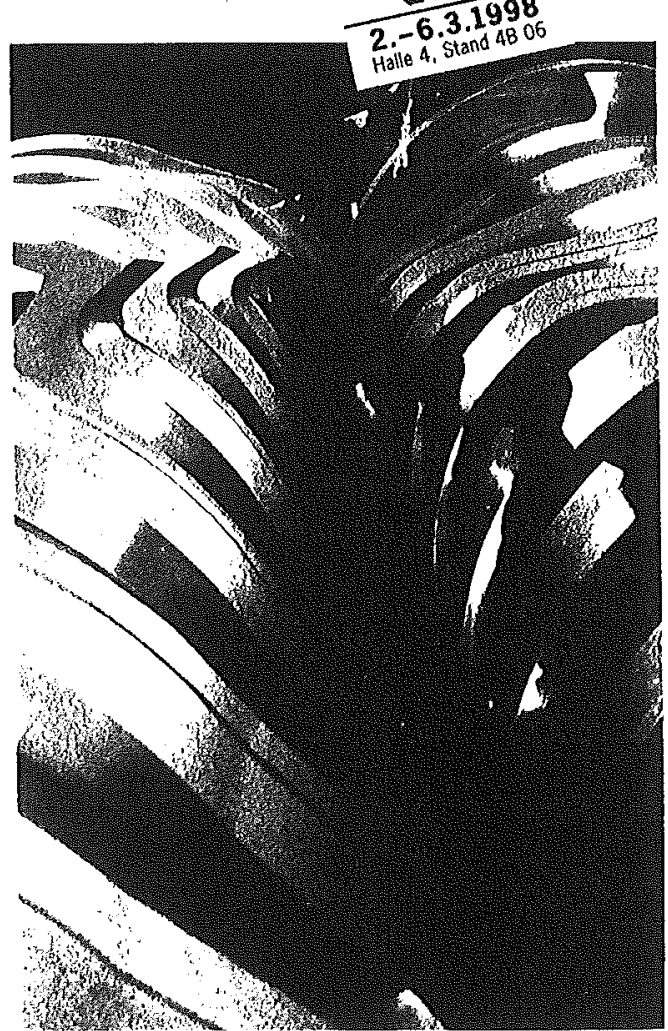

Risurscheren 


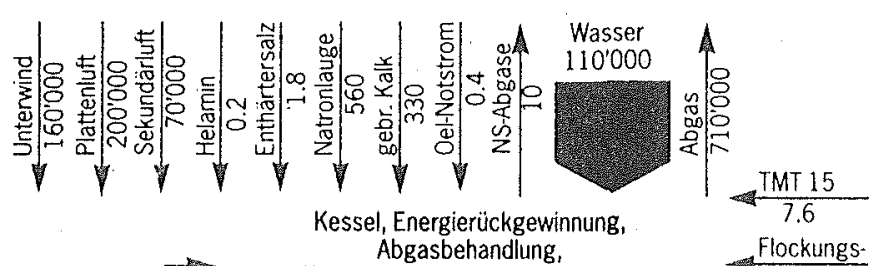

Hilfsmittelzubereitung und verteilung, mittel 0.3 Aschewaschung, Abwasserreinigung

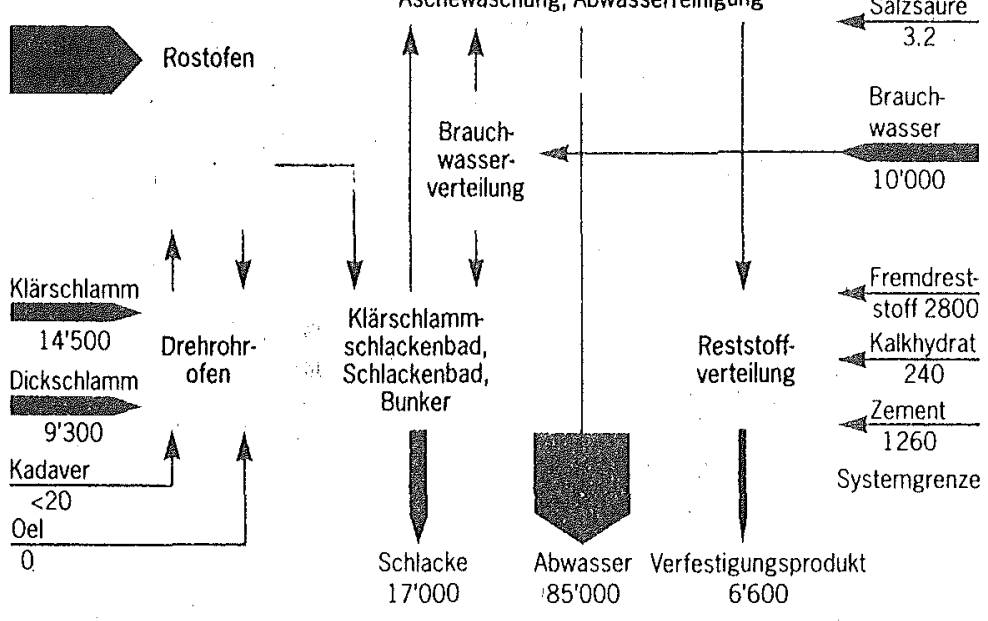

Abbildung 3.1.1:

Güterfluß durch die

Müllverbrennungsan-

lage Oftringen für

das Jahr 1996 in

Tonnen/Jahr. Gas-

flusse und Flüsse in-

nerhalb des Systems werden nicht wert-

proportional darge-

stelit. Vertrauensbe-

reiche: Gasfluisse

$15 \%$, andere Flüsse

kleiner als $5 \%$.

SA: Siedlungsabfall

Abbildung 3.2.1

Zinkkonzentrationen

in den einzelnen

Schlackenproben aus

dem Bunker und in

den Mischproben an

verschiedenen Tagen

MW: Mittelwert

Schraffierter

Bereich: Vertrauens-

bereich des Mittel-

wertes

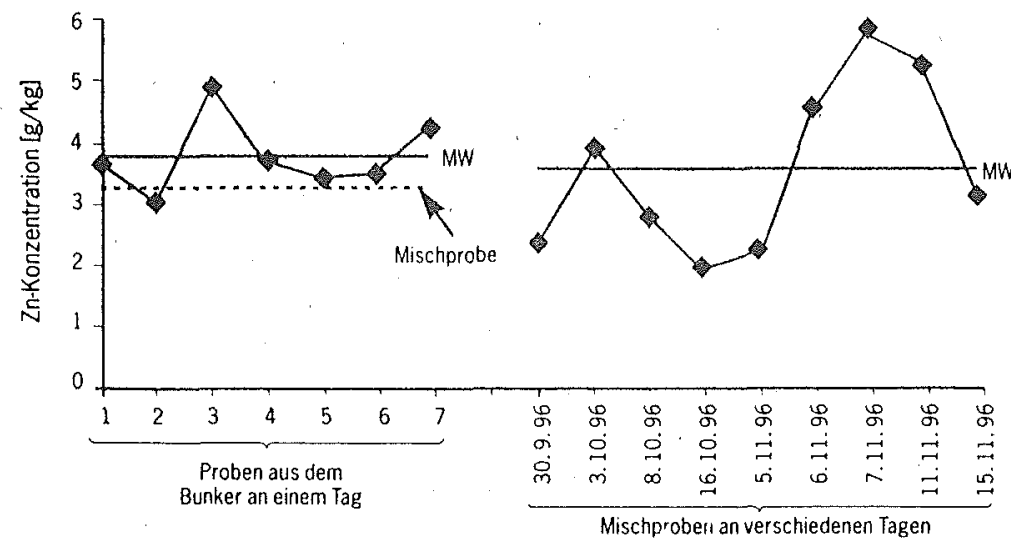

Die Proben werden bei $110^{\circ} \mathrm{C}$ getrocknet und anschlieBend gemahlen.

In der Vorbereitungsphase werden außerdem bei zwei verschiedenen Verfestigungen 3 bzw. 4 Einzelproben (Mischprobe aus einer Mulde) entnommeni, um trationen in der Mischprobe zu vergleichen.

\subsubsection{Abwasser}

Aus dem letzten Abwassertank vor der Einleitung in die ARA wird viermal ca. 11 Probe im Abstand von 15 Minuten entnommen. Die Proben werden durchmischt, eine Teilmenge von ca. 11 in eine Flasche abgeKühlschrank aufbewahrt.

\subsubsection{Chemische Analysen} dem wellenlängendispersiven Röntgenfluoreszenzspektrometer ARL 8410 der Firma ARL analysiert. Dazu wird ein eigenes Eich- und Kalibrierprogramm (BELLA 1), das für die Rückstände der Müllverbrennungsanlage entwickelt wurde, verwendet. Die Konzentrationen der Elemente $C$ und $S$ werden mit dem CNS-Analyzer CNS 1500 der Firma Carlo Erba bestimmt, Chlor nach dem Aufschluß nach Wurzschmitt [13] mit lonensensitiver Elektrodeund die Konzentrationen der Schwermetalle nach dem Königswasseraufschluß mit einem Atomabsorptionspektrometer.

Für die Konzentrationsbestimmungen in den flüssigen Proben werden Plasma-Emissionsspektrometrie (ICP-AES), Atomabsorptionsspektrometrie (AAS) und Ionenchromatographie eingesetzt.

\subsection{Berechnuing der Transferkoeffizienten}

Der Transferkoeffizient $\mathrm{k}_{\mathrm{ib}}=\mathrm{b} / \mathrm{a}$ bezeichnet die Fraktion des gesamten in den Prozeß eingeführten Stoffes i, die in das Gut b transferiert wird [3]. Dabei bezeichnet a den totalen Input. In dieser Arbeit werden auch die kWerte für Inputgüter angegeben und auch als Transferkoeffizienten bezeichnet. Sie werden mit der Einführung von einem fiktiven Verteilungsprozess zwischen allen Herkunftsprozessen der Anlage und den Prozessen in der Systemgrenze berechnet und zeigen die Anteile der verschiedenen lnputgüter am gesamten Input. die Konzentrationen in diesen Proben mit den Konzenfüllt, mit konz. Salpetersäure angesäuert und in einem

Die Elemente $\mathrm{Na}, \mathrm{Mg}, \mathrm{Al}$, Si, P, K und Ca werden mit

\subsection{Fehlerabschätzung}

Die folgenden Formeln wurden für die Abschätzung der Zufallsfehler von Flüssen, Konżentrationen und Transferkoeffizienten verwendet [14]:

Standardabweichung

$$
S=\sqrt{\frac{\sum\left(x-\bar{x}^{2}\right)}{n-1}}
$$
$\mathrm{x}$ : Einzelwerte
$\overline{\mathrm{x}}$ : Mittelwert
n: Anzahl Meßwerte

Standardabweichung von Mehrfachbestimmungen

$$
S_{M+1}=\sqrt{\frac{\sum_{i=1}^{n}}{n} \frac{\sum_{i=1}^{m}\left(x_{i j}-\bar{x}\right)^{2}}{(m-1)}}
$$

$x_{i j}$ :j-te Bestimmung an der i-ten Untersuchungseinheit

$\mathrm{m}$ :Anzahl der Mehrfachbestimmungen

$\mathrm{n}$ : Anzahl der Untersuchungseinheiten (z.B. Proben) - Standardabweichung innerhalb der Messreihen (gewichtete Standardabweichung)

$$
s_{i n}=\sqrt{\frac{s_{1}^{2}\left(n_{1}-1\right)+s_{2}^{2}\left(n_{2}-1\right)+\ldots+s_{k}^{2}\left(n_{k}-1\right)}{n-k}}
$$

\section{k: Anzahl der Meßreihen}

Vertrauensbereich des Mittelwertes

$$
V B=\bar{x} \pm t_{|n-1, \alpha|} \cdot \frac{s}{n}
$$


t: Faktor der Studentverteilung

$\alpha$ : Irrtumswahrscheinlichkeit (hier $\alpha=0.05$ gewählt)

Relativer Vertrauensbereich des Mittelwertes

$$
V_{\mathrm{rel}}=\frac{t_{(n-1, \alpha)} \cdot \frac{s}{n}}{\bar{x}}
$$

Fehlerfortpflanzung nach Gauss

$$
\Delta F\left(x_{1}, x_{2}, \ldots\right)=\sqrt{\left(\frac{\partial F}{\partial x_{1}}\right)^{2}\left(\Delta x_{1}\right)^{2}+\left(\frac{\partial F}{\partial x_{2}}\right)^{2}\left(\Delta x_{2}\right)^{2}+\ldots}
$$

Im folgenden wird der relative Vertrauensbereich des Mittelwertes, der durch die Standardabweichungen der Laborbestimmungen berechnet wird, als Laborfehler bezeichnet.

\subsection{Auswertung der Daten}

Für die Auswertung der Daten muß eine Datenbank aufgebaut werden. Sie kann mit Personal Computer tauglicher Software (z.B. EXCEL, ACCESS) erstellt und betrieben werden. Für die Güterflüsse ist ein Tag und für die Stoffflüsse ist eine Woche der kleinste Zeitabschnitt für die Dateneingabe. Im Auswertungsteil werden die Berechnungen durchgeführt und Fehler aufgefangen. Die Auswertungen werden für eine gewünschte Bilanzierungsperiode durchgeführt, um die zeitlichen Variationen feststellen zu können. Im Ausgabeteil werden die Güterflüsse, die Konzentrationen in Funktion der Zeit und die Stoffflüsse in der gewünschten Zeitperiode als Abbildungen und Tabellen veranschaulicht. In Stoffflußdiagrammen werden die Mittelwerte und Vertrauensbereiche der Flüsse, Konzentrationen und Transferkoeffizienten angegeben, sofern sie signifikant sind. Diese Art der Datenauswertung, Visualisierung und Simulation kann z.B. mit SIMBOX durcgheführt werden [3].

\section{Resultate und Diskussion}

\subsection{Güterflüsse}

Die Güterflüsse wurden ab Anfang 1996 kontinuierlich erfaßt und ausgewertet. Abbildung 3.1.1 zeigt die Güterflüsse im Jahre 1996. Die Prozesse wurden soweit aggregiert, damit einige Informationen für die Herkunfts- und Zielprozesse der Güter erhalten werden können, obwohl die inneren Flüsse nicht erfaßt werden. Die Inpu - und Outputpfeile wurden .vertproportional dargescellt, außer für die Zuluftflüsse und den Abgasfluß. Die Flüsse innerhalb des Systems wurden nicht bestimmt und deshalb nicht wertproportional dargestellt. Die Zuluft und das Abgas machen die größten Güterflüsse aus (430.000 t/a, bzw. 710.000t/a). Das mengenmäßig zweitwichtigste Gut ist das Wasser; ca. $110.000 \mathrm{t} / \mathrm{a}$ Leitungswasser und ca. $10.000 \mathrm{t} / \mathrm{a}$ Brauchwasser aus der ARA fließen in die Anlage. Die Anlage verbrennt 59.800 t/a Siedlungsabfall und ca. $24^{\prime} 000$ t/a Klärschlamm und Dickschlamm. Bezüglich der Trockensubstanz ist das Verhältnis Siedlungsabfall zu Schlamm ca. 8 zu 1. Der Schlackenanfall (Müllschlacke + Klärschlammschlacke) ist ca. 17.000 t/a. Die jährliche Kesselstaub-, Elektrofilter- und Abwasser-

\begin{tabular}{|c|c|c|c|c|}
\hline Element & $\begin{array}{l}\text { Mittelwert der } \\
\text { Tagesmisch- } \\
\text { proben } \\
{[\mathrm{g} / \mathrm{kg}]}\end{array}$ & $\begin{array}{l}\text { Relativer } \\
\text { Vertrauensbereich } \\
\text { des Mittelwertes } \\
\text { der Tagesmisch- } \\
\text { proben } \\
{[\%]}\end{array}$ & Laburfehler & $\begin{array}{l}\text { Relativer } \\
\text { Vertrauensbereich } \\
\text { des Mittelwertes } \\
\text { der Proben aus } \\
\text { dem Bunker } \\
{[\%]}\end{array}$ \\
\hline
\end{tabular}
schlammmengen betragen ca. 2.280 t/a (6600-1260-240-

\begin{tabular}{lrrll}
\hline \multicolumn{2}{l}{ Metalle und Metalloide } & & & \\
$\mathrm{Na}$ & 17 & 12 & 4 & 29 \\
$\mathrm{Mg}$ & 14 & 5 & 2 & 8 \\
$\mathrm{Al}$ & 54 & 6 & 3 & 6 \\
$\mathrm{Si}$ & 197 & 5 & 0.4 & 6 \\
$\mathrm{~K}$ & 10 & 5 & 3 & 4 \\
$\mathrm{Ca}$ & 122 & 4 & 1 & 6 \\
\hline
\end{tabular}

Nichtmetalle

$\begin{array}{rrrrr}\mathrm{C} & 24 & 12 & 5 & 41 \\ \mathrm{P} & 11 & 24 & 3 & 38 \\ \mathrm{~S} & 2.8 & 19 & 14 & 41 \\ \mathrm{C} & 3.9 & 7 & 9 & 17\end{array}$

Schwermetalle

\begin{tabular}{lllll}
$\mathrm{Cu}$ & 3.1 & 29 & 9 & 48 \\
$\mathrm{Zn}$ & 3.6 & 30 & 4 & 15 \\
$\mathrm{Cd}$ & 0.0075 & 88 & 7 & 67 \\
$\mathrm{~Pb}$ & 1.3 & 34 & 7 & 21 \\
\hline
\end{tabular}

Tabelle 3.2.1:

Mittlere Konzentra.

tionen der Stoffbuch haltungselemente in Tagesmischproben der Schlacke und ein Vergleich verschiedener Streuungen

- Laborfehler werden durch Wie derholungen der chemischen Analysen bestimmt

- Relativer Vertrauensbereich des Mittelwertes der Tagesmischproben ist ein Hinweis für die zeitlichen $\mathrm{Va}$ riationen inklusive Probenahme- und Laborfehler

- Relativer Vertrauensbereich des Mittelwertes der Proben aus dem Bunker ist eine obere Grenze für die Summe der Probenahmefehler und Laborfehler
2820). Diese Menge wird mit ungefähr der gleichen Menge Fremdreststoff ( 2820 t/a) vermischt und mit Zement und Kalkhydrat verfestigt. Es entsteht außerdem eine erhebliche Menge Abwasser (85.000 t/a), das in die ARA eingeleitet wird. Zement, Kalkhydrat, gebrannter Kalk und Natronlauge sind die mengenmäßig wichtigsten Betriebshilfsmittel. Die anderen Betriebsmittel machen nur einen sehr kleinen Anteil der Güterflüsse aus.

\subsection{Stoffflüsse}

1996 wurden neben der kontinuierlichen Erfassung der Güterflüsse auch zahlreiche Konzentrationsmessungen durchgeführt, um die Probenahmen optimieren zu können. In Abschnitt 3.2.1 werden die statistischen Unsicherheiten und zeitliche Variationen der Konzentrationen in der Schlacke und in den Verfestigungsprodukten angegeben. Basierend auf diesen Messungen können die Stoffflüsse für das Jahr 1996 grob abgeschätzt werden. In Abschnitt 3.2.3 werden die Abschätzungen für die Chlor- und Zinkflüsse vorgestellt.

Ab Anfang 1997 wurden die Güterflüsse weiterhin kontinuierlich erfaßt und zusätzlich auch die Inputund Outputgüter gemäß Tabelle 2.4 .1 kontinuierlich beprobt, analysiert (kontinuierliche Stoffbuchhaltung) und ausgewertet. In Abschnitt 3.2.4, werden exemplarisch nur die Zinkkonzentrationen in den regelmäßig beprobten Gütern in Funktion der Zeit und die Zinkflüsse zwischen Januar 1997 und April 1997 vorgestellt.

3.2.1 Statistische Unsicherheiten und zeitliche Variationen der Konzentrationen in der Schlacke

Als zeitliche Variationen werden die durch Veränderungen der Menge und Zusammensetzung in Input und die verfahrenstechnischen Änderungen bedingten Konzentrationsänderungen bezeichnet. In der 


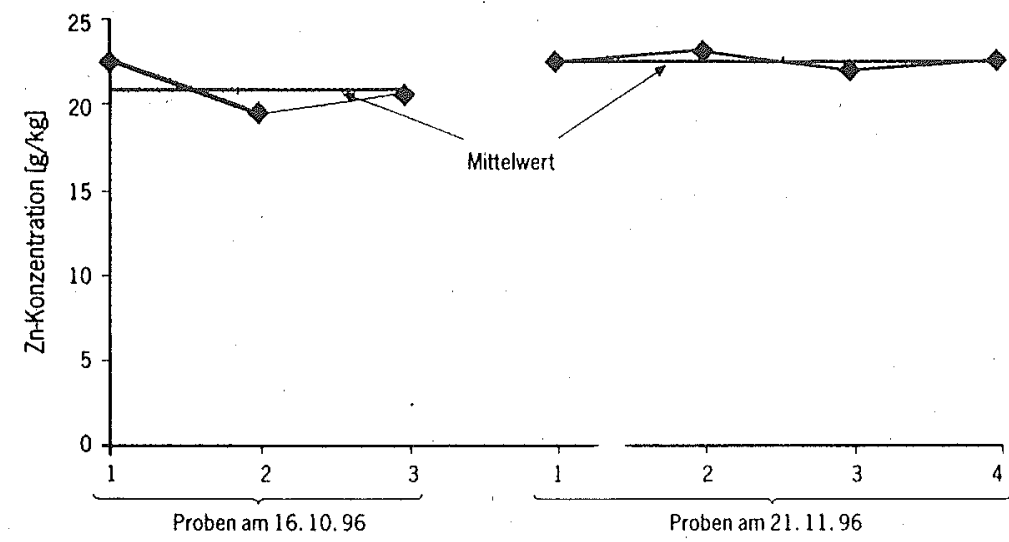

Abbildung 3.2.2

Zinkkonzentrationen

in den Verfestigungs

produkten, die jeweils

aus einer Mulde als

Mischprobe ent-

nommen wurden

Schraffierter

Bereich: Vertrauens-

bereich des Mittel-

wertes

vorliegenden Arbeit wird dies am Beispiel Zink exemplarisch veranschaulicht. Der Grund für die Wahl von Zink wird in Abschnitt 3.2.4 angegeben. Abbildung 3.2.1 zeigt die Zinkkonzentrationen in den einzelnen Schlackenproben aus dem Bunker und in den Mischproben an verschiedenen Tagen. Die Streuung der Proben aus dem Bunker kann auf die Probenahmeund Laborfehler hinweisen. Es ist zu erwarten, dass die Summe der Probenahmefehler und Laborfehler kleiner ist als der Vertrauensbereich des Mittelwertes der Proben aus dem Bunker. Folglich sollte der Wert für die Mischprobe im Vertrauensbereich des Mittelwertes

Tabelle 3.2.2:

Mittlere Konzentrationen der Stoffbuchhaltungelemente in den Verfestigungsprodukten und ein Vergleich verschiedener Streuungen

- Laborfehler werden durch Wiederholungen der chemischen Analysen bestimint

- Relative Differenz zwischen Tagesmittelwerten und Mittelwert aller Proben ist ein Hinweis für die zeitlichen Variationen inklusive Probenahme- und Laborfehler (nur zwei Werte)

- Gewichteter relativer Vertrauensbereich des Mittelwertes aller Proben ist Hinweis für die Summe der maximalen Probenahmefehler und Laborfehler

\begin{tabular}{|c|c|c|c|c|}
\hline Element & $\begin{array}{l}\text { Mittelwert } \\
\text { aller Proben }\end{array}$ & $\begin{array}{l}\text { Relative Differenz } \\
\text { zwischen Tages- } \\
\text { mittelwerten und } \\
\text { Mittelwert aller } \\
\text { Proben } \\
{[\%]}\end{array}$ & Laborfehter & $\begin{array}{l}\text { Gewichteter } \\
\text { relativer } \\
\text { Vertrauensbereich } \\
\text { des Mittelwertes } \\
\text { aller Proben } \\
{[\%]}\end{array}$ \\
\hline
\end{tabular}

\begin{tabular}{lrrrr}
\hline \multicolumn{2}{l}{ Metalle und Metalloide } & & & \\
$\mathrm{Na}$ & 8 & 14 & 1 & 10 \\
$\mathrm{Mg}$ & 11 & 4 & 0.4 & 2 \\
$\mathrm{Al}$ & 40 & 6 & 1 & 5 \\
$\mathrm{Si}$ & 100 & 4 & 1 & 3 \\
$\mathrm{~K}$ & 7 & 17 & 2 & 5 \\
$\mathrm{Ca}$ & 275 & 4 & 1 & 3 \\
\hline
\end{tabular}

Nichtmetalle

$\begin{array}{lcrrr}C & 11 & 10 & 10 & 10 \\ P & 4 & 12 & 1 & 7 \\ S & 19 & 43 & 21 & 26 \\ C l & 3.5 & 4 & 4 & 7\end{array}$

Schwermetalle

$\begin{array}{lcrrrl}\mathrm{Cu} & 1.0 & 4 & 3 & 9 & \\ \mathrm{Zn} & 22 & 4 & 4 & 5 & \\ \mathrm{Cd} & 0.32 & 23 & 2 & 10 \\ \mathrm{~Pb} & 7.1 & 3 & - & 4 & 3\end{array}$

der Einzelproben aus dem Bunker liegen. Dies ist der Fall für Zink (Abbildung 3.2.1). Der Vertrauensbereich des Mittelwertes der Tagesmischproben ist ein Mass für die zeitlichen Variationen und beinhaltet auch die Probenahme- und Laborfehler. Die Einzelwerte aus dem Bunker sind zwischen $3 \mathrm{~g} / \mathrm{kg}$ und $5 \mathrm{~g} / \mathrm{kg}$. Die zeitlichen Variationen der Mischproben an verschiedenen Tagen ist höher. Sie variieren von $2 \mathrm{~g} / \mathrm{kg}$ bis $6 \mathrm{~g} / \mathrm{kg}$. Diese Resultate zeigen, dass eine Messung durch eine Mischprobe an einem Tag bis zu 100\% vom Mittelwert von 9 Tagen abweichen kann. Wenn statt eine Mischprobe nur eine Einzelprobe entnommen wird, kann die Abweichung noch höher werden.

Tabelle 3.2.1 zeigt die mittleren Konzentrationen der Stoffbuchhaltungselemente in Tagesmischproben der Schlacke und einen Vergleich verschiedener statistischer Unsicherheiten und zeitlicher Variationen. Die zeitlichen Variationen der Konzentrationen der Metalle und Metalloide sind nicht sehr hoch; die relativen Vertrauensbereiche des Mittelwertes der Tagesmischproben sind kleiner als $12 \%$. Die Heterogenität im Bunker ist auch klein; der relative Vertrauensbereich des Mittelwertes der Tagesmischproben ist tiefer als $10 \%$. Natrium ist dabei eine Ausnahme; die Heterogenität im Bunker bezüglich Natrium ist höher als die Heterogenität bezüglich anderer Elemente. Die Laborfehler sind kleiner als 5\%.

Die relativen Vertrauensbereiche der Konzentrationen von Nichtmetallen sind höher als bei den Metallen und Metalloiden. Wie bei den Metallen und Metalloiden sind jedoch die relativen Vertrauensbereiche des Mittelwertes der Tagesmischproben tiefer als die rela tiven Vertrauensbereiche des Mittelwertes der Proben aus dem Bunker. Die Laborfehler sind noch tiefer (Schwefel ist eine Ausnahme). Diese Resultate zeigen, dass die Elemente $C$ und $P$ sehr heterogen im Bunker verteilt sind. Dies ist zu erwarten, da die Konzentrationen dieser Elemente in der Klärschlammschlacke höher sind als in der Müllschlacke [17]. Bei Chlor und Schwefel sind Laborfehler in der gleichen Grössenordnung wie die zeitlichen Variationen. Der relative Vertrauensbereich des Mittelwertes der Chlorkonzentrationen in den Tagesmischproben ist jedoch klein (7\%). Eine Analyse von Chlor in den einzelnen Tagesmischproben ist deswegen nicht notwendig. Das gleiche gilt auch für Schwefel, da der Laborfehler zu hoch ist.

Die Schwermetalle $\mathrm{Cu}, \mathrm{Zn}, \mathrm{Cd}$ und $\mathrm{Pb}$ zeigen höhere zeitli re Variationen. Die Laborfehler sind tiefer als $10 \%$ fur diese Elemente. Die Probenahmefehler sind bei der Bestimmung der Konzentrationen von Kupfer und Cadmium wahrscheinlich viel höher als diejenigen für Zink und Blei, da die Heterogenität im Bunker hezüglich Kupfer und Cadmium viel höher ist als die Heterogenität bezüglich Zink und Blei (relativer Vertrauensbereich $48 \%$ bzw. $67 \%$ für Kupfer bzw. Cadmium und $15 \%$ bzw. $21 \%$ für Zink bzw. Blei). Aus diesem Grund sind Zink und Blei besser geeignet für die Feststellung der kurzzeitigen zeitlichen Variationen (Wochen bis Monate) als Kupfer und Cadmium.

3.2.2 Statistische Unsicherheiten und zeitliche Variationen der Konzentrationen in den Verfestigungsprodukten

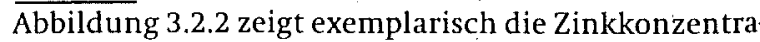


tionen in den Verfestigungsprodukten, die jeweils aus einer Mulde entnommen wurden. Die Proben wurden an zwei verschiedenen Verfestigungstagen genommen. Die Abweichungen vom Mittelwert innerhalb der Mulden an einem Tag sind nicht gross (Differenz Maximalund Minimalwert: $<20 \% 1$. Der Vertrauensbereich des Mittelwertes liegt auch in einem engen Bereich.

Tabelle 3.2.2 zeigt die mittleren Konzentrationen der Stoffbuch haltungelemente in den Verfestigungsprodukten und einen Vergleich verschiedener statistischen Unsicherheiten und zeitlicher Variationen. Die Konzentrationen der Metalle und Metalloide schwanken nicht stark in den Verfestigungsprodukten. Der gewichtete relative Vertrauensbereich des Mittelwertes aller Proben ist tiefer als $10 \%$. Die zeitlichen Variationen sind höher (nur zwei Werte). Die Laborfehler sind kleiner als $2 \%$ bezüglich des Mittelwertes.

Die Nichtmetalle und Schwermetalle zeigen das gleiche Bild; der gewichtete relative Vertrauensbereich des Mittelwertes aller Proben ist tiefer als 10\%. Schwefel ist eine Ausnahme, da der Laborfehler zu hoch ist (21\%). Ausserdem zeigen die Cadmiumkonzentrationen grössere Abweichungen vom Mittelwert als die anderen Elemente.

Diese Resultate zeigen, dass die Verfestigungsprodukte im allgemeinen homogener sind als die Schlacken. Das ist zu erwarten, da sie während 2 bis 4 Wochen gesammelt und dann während der Verfestigung homogenisiert werden. Weil sie auch kumulative Proben sind, sind sie repräsentativ für die Sammelperiode. Schlacken sind dagegen nur repräsentativ für den Probentag. Da neben dem hohen Informationswert dieser Proben auch ihre Aufbereitung mit einem relativ kleinen Aufwand verbunden ist, soll in der MVA-Stoffouch haltung jede Verfestigungscharge beprobt werden.

3.2.3 Abschätzung der Chlor-und Zinkflüsse für das Jahr 1996 Stellvertretend für die 14 Elemente sollen die Chlorund Zinkflüsse vorgestellt werden. Abbildung 3.2.3. zeigt den Chlorfluss durch die Anlage für das Jahr 1996 in t/a. Der Gesamtinput in die Anlage beträgt ca. 510 t/a. Der Siedlungsabfall trägt $96 \%$ zum Gesamtinput bei. Weitere $2 \%$ kommen vom Fremdreststoff. Der Anteil dés Klärschlamms und Dickschlamms ist kleiner als 1\%. Der Rest kommt durch die Betriebsmittel in die Anlage. $86 \%$ des Chlors verlassen das System durch das Abu'asser. 10\% durch die Schlar':e und $4 "$ durch das Verfejtigungsprodukt. Weniger ais $1 \%$ des Chloroutputs erfolgt durch das Abgas in die Atmosphäre. Daraus errechnet sich ein Wirkungsgrad von mehr als 99\% für die Chlorabscheidung aus dem Rohwas im Gasreinigungssystem der Müllverbrennungsanlage Oftringen. Etwa die Hälfte des im Verfestigungsprodukt befindlichen Chlors kommt aus der MVA Ofuringen, die andere Hälfte aus anderen Müllverbrennungsanlagen durch das Fremdprodukt. Insgesamt niessen also ca. $86 \%$ des Chlors durch die ARA in die Vorhuter, $14 \%$ in die Deponien und weniger als $1 \%$ in die Atmosphäre (vgl. Abbildung 2.2.1).

Abbildung 3.2.4. zeigt den Zinkfluss durch die Anlige fiir das Jahr 1996 in t/a. Der Gesamtimput in die Anlagge beträgt ca. 100 t/a. Der Siedlungsabfall trägt
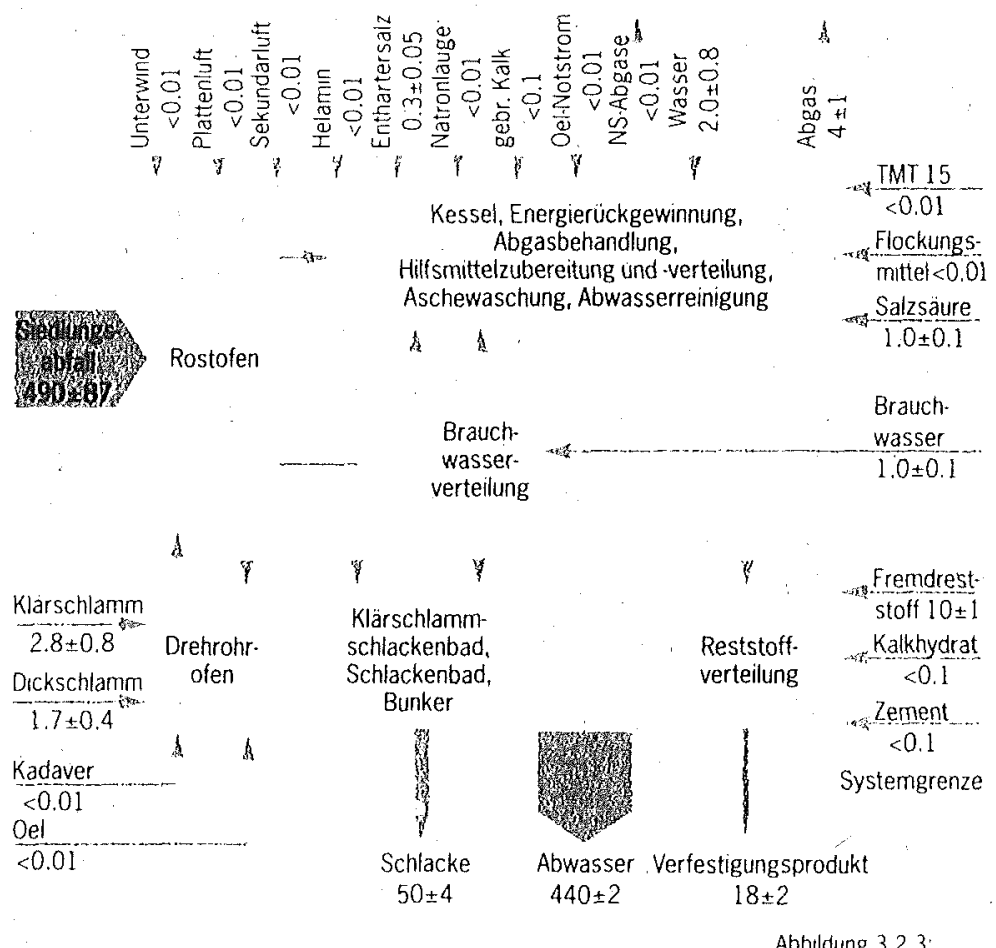

$61 \%$ zum Gesamtinput bei. Weitere $34 \%$ kommen von Fremdreststoff. Der Anteil des Klärschlamms und Dickschlamms ist ca. $5 \%$. Mit den Betriebsmitteln kommen weniger als $1 \%$ des Zinks in die Anilage.

$67 \%$ des Zinks verlassen das System durch das Verfestigungsprodukt und 33\% durch die Schlacke. Weniger als $0.1 \%$ des Zinkoutputs erfolgt durch das Abgas in die Atmosphäre. Daraus errechnet sich ein Wirkungsgrad von mehr als $99 \%$ für die Zinkabscheidung aus dem Rohgas im Gasreinigungssystem der Müllverbrennungsanlage Oftringen. Nur ein sehr kleiner Teil des Zinkoutputs befindet sich im Abwasser. Ungefähr die Hälfte des im Verfestigungsprodukt befindlichen

Abbildung 3.2.3: Müllverbrennungsanlage Oftringen für das Jahr 1996 in Tonnen/Jahr. Flüsse innerhalb des Systems werden nicht wertproportio. nal dargestelit.

Abbuldung 3.2.4: Zinkfluss durch die Müllverbrennungsanlage Oftringen für das Jahr 1996 in Tonnen/Jahr.

Flüsse innerhalb des Systems werden nicht wertproportio nal dargestellt. FRST:Farbreststof

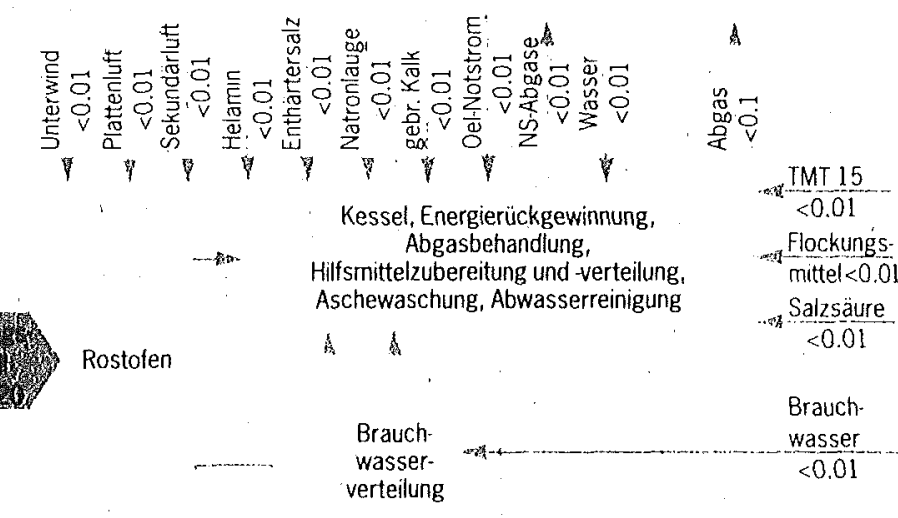

\begin{tabular}{|c|c|c|c|c|c|}
\hline Klärcrblamm & s & $y$ & & $p$ & 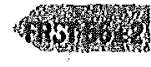 \\
\hline $\begin{array}{c}6.5 \pm 5.0 \\
\text { Dickschlamm }\end{array}$ & $\begin{array}{l}\text { Drehrohr- } \\
\text { ofen }\end{array}$ & $\begin{array}{c}\text { Klärschlamm- } \\
\text { schlackenbad, } \\
\text { Schlackenbad, } \\
\text { Bunker }\end{array}$ & i & $\begin{array}{l}\text { Reststoff- } \\
\text { verteilung }\end{array}$ & $\begin{array}{l}-\frac{\text { Kalkhydrat }}{<0.01} \\
\text { Zement }\end{array}$ \\
\hline $\begin{array}{l}2.4 \pm 1.1 \\
\text { Kadaver } \\
<0.01 \\
0 \text { el }\end{array}$ & 点 & 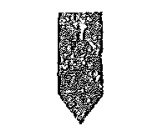 & 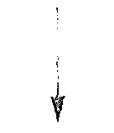 & & $\begin{array}{c}<0.2 \\
\text { Systemgrenze }\end{array}$ \\
\hline$<0.01$ & & $\begin{array}{c}\text { Schlacke } \\
55 \pm 24\end{array}$ & $\begin{array}{c}\text { Abwasser } \\
<0.02\end{array}$ & $\begin{array}{r}\text { Verfestigungsp } \\
110 \pm 3\end{array}$ & dukt \\
\hline
\end{tabular}


Zinks kommt aus der MVA Oftringen, die andere Hälfte aus anderen Müllverbrennungsanlagen durch das Fremdprodukt. Insgesamt gelangen also mehr als $99 \%$ des Zinks in die Deponien und weniger als $1 \%$ in die Vorfluter und in die Atmosphäre (vgl. Abbildung 2.2.1).

\subsubsection{Kontinuierlich bestimmte Stoffflüsse zwischen Januar} 1997 und April 1997

Aus den Resultaten, die in den Abschnitten 3.2.1 und 3.2 .2 vorgestellt worden sind, ist ersichtlich, dass die Bestimmung der Konzentrationen der Metalle und Metalloide in den Einzelproben nicht unbedingt notwendig ist, da die zeiclichen Variationen dieser Elemente sowohl in der Schlacke als auch in den Verfestigungsprodukten ohnehin klein sind $(<12 \%)$. Nichtmetalle und Schwermetalle sind im allgemeinen besser geeignet für die Bestimmung der kurzzeitigen Varia-
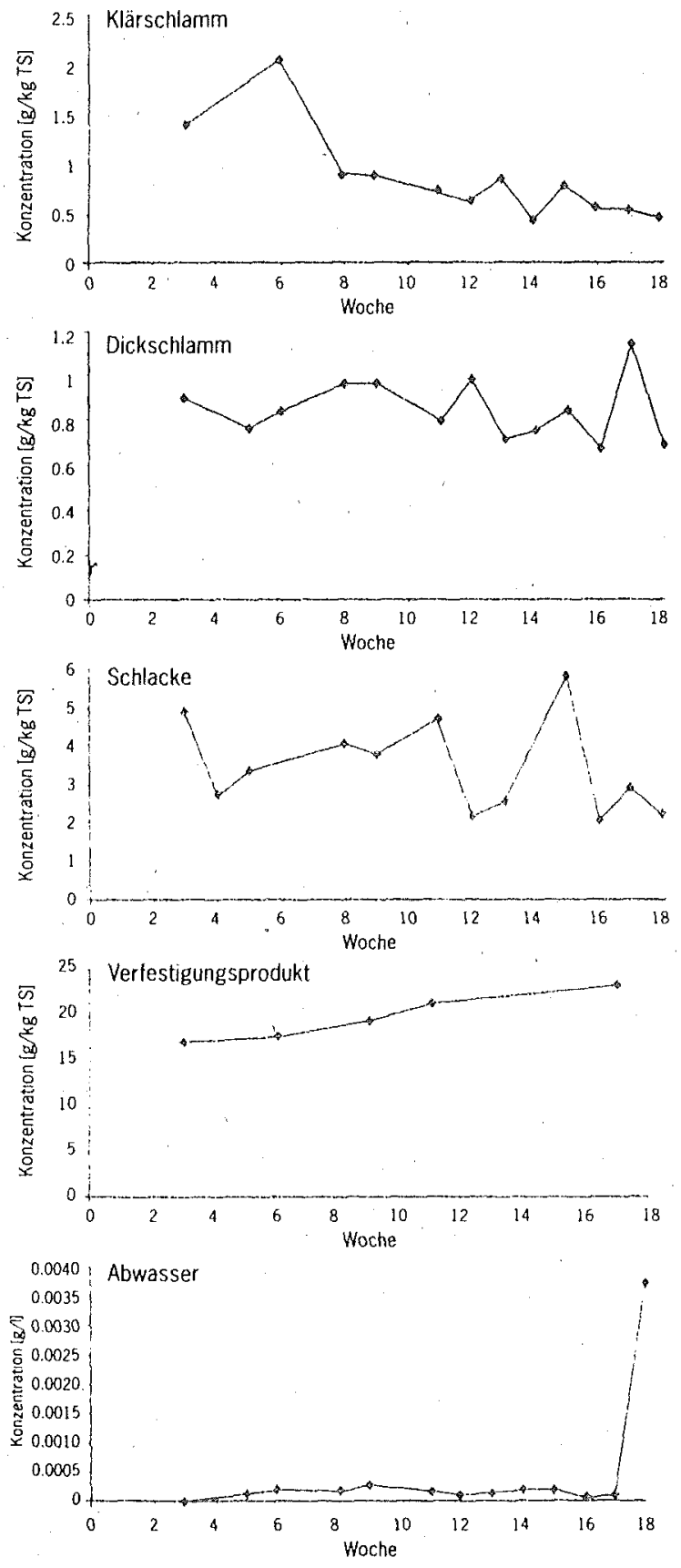

tionen (Wochen bis Monate) der Inputeigenschaften und der Prozessbedingungen. Zink ist gewählt worden da im Ofen ca.die Hälfte von Zink ins Rohgas und die andere Hälfte in die Schlacke transferiert wird. Dieser Transfer reagiert empfindlich auf die Inputeigenschaften und Prozessbedingungen [11|. Dadurch können Hinweise sowohl über die Veränderungen der Inputqualität als auch über die Prozessveränderungen erhalten werden. Ausserdem ist Zink aus probenahmetechnischen und chemisch-analytischen Gründen sehr gut geeignet für die Feststellung der kurzzeitigen Variationen der Konzentrationen (Wochen bis Monate) in dei Schlacke und in den Verfestigungsprodukten (Abschnitt 3.2.1 und 3.2.2). Der Aufwand für die Bestimmung der Konzentrationen weiterer Elemente ist jedoch klein. Kupfer wurde zum Beispiel zusätzlich gewählt, da die Aufsichtsbehörde der MVA Oftringen hin und wieder Angaben über Kupferkonzentrationen in der Schlacke wünscht und dieser Wunsch mit einem kleinen zusätzlichen Aufwand sehr leicht erfüllt werden kann. Zeitliche Variationen der Flüsse von weiteren Elementen können auch später bestimmt werden, da die Einzelproben aufbewahrt werden müssen.

\subsubsection{Illustration von Anwendungen der Stoffbuchhaltung 3.2.5.1 Input/Output-Controlling}

Abbildung 3.2.5. zeigt den zeitlichen Konzentrationsverlauf des Zinks in den regelmässig beprobten Gütern. Die Zinkkonzentration im Klärschlamm zeigt eine Abnahme. Die Laborfehler sind vernachlässigbar $(0.2 \mathrm{~g} / \mathrm{kg}$ TS). Die Konzentrationen im Dickschlamm schwanken zwischen $0.7 \mathrm{~g} / \mathrm{kg}$ TS und $1.1 \mathrm{~g} / \mathrm{kg}$ TS (TS: Trockensubstanz). Die beobachteten Abweichungen vom Mittelwert können von zeitlichen Variationen, Probenahmefehlern und Labor fehlern $(0.1 \mathrm{~g} / \mathrm{kg} \mathrm{TS})$ herrühren. Die Konzentrationen in der Laborprobe der Schlacke variieren zwischen $2.0 \mathrm{~g} / \mathrm{kg}$ TS und $5.8 \mathrm{~g} / \mathrm{kg}$ TS. Der Laborfehler ist kleiner als $0.7 \mathrm{~g} / \mathrm{kg}$ TS. Die Konzentrationsunterschiede können von den Probenahmefehlern und von den zeitlichen Variationen herrühren. Bei den Flussberechnungen wird der Mittelwert dieser Konzentrationen unter Berücksichtigung der abgeschätzten Konzentrationen im Grobgut und im Siebrest korrigiert (Abschnitt 2.6.4). Der Konzentrationsverlauf im Verfestigungsprodukt zeigt eine $\mathrm{Zu}$ nahme. Die Konzentration im Abwasser ist im allgemeinen kleiner als $0.5 \mathrm{mg} / \mathrm{l}$. In der Woche 18 wurde jedoch eine Konzentration von $3.7 \mathrm{mg} / 1$ gemessen. Dieser Wert ist nicht durch Probenahmefehler oder chemisch-analytische Fehler entstanden und weist auf eine zeitweilig nicht funktionierende Abwasseraufbereitung hin. Dies ist ein Beispiel, wie die Methode für das Controlling der Produktequalitäten eingesetzt werden kann.

Im allgemeinen liefern die Konzentrationsverläufe erste Hinweise über den Input und die Prozesse in der MVA. Um statistisch signifikante Aussagen machen zu können, müssen noch Stoffflüsse und Transferkoeffizienten in Betracht gezogen werden. Abbildung 3.2.6 zeigt die wertproportionale Darstellung der Input- und Outputflüsse des Zinks in den ersten 18 Wochen.

Der Gesamtinput an Zink in den ersten 18 Wochen 
beträgt 56 Tomnen. Auf das ganze Jahi hochgerechnet würden ca. 160 Tonnen Zink pro Jahr in die Anlage fliessen. Dies ist die genau gleiche Menge Zink, die $1996 \mathrm{in}$ die MVA eingeliefert worden ist $160 \mathrm{t} / \mathrm{a}, \mathrm{vgl} . \mathrm{Ab}$ bildung 3.2.41. 76" des Inputs erfolgen durch den Siedlungsabfall $(42 \pm 10 t) .22^{\prime \prime}$ werden im Frendreststoff eingeführt $(12 \pm 1 \mathrm{t})$. Weniger als $3^{\prime \prime \prime}$ des Zinkinputs erfolgt über Klärschlamm und Dickschlamm. Der Input durch Betriebs- und Hilfsstoffe ist vernachlässigbar.

Das Verfestigungsprodukt trägt 59"i zum Zinkoutput bei $(33 \pm 5 \mathrm{t}) .40 \%$ des Zinks verlassen das System via Schlacke in der Bilanzperiode $(23 \pm 9 t)$. Die Anteile des Abwassers und der Abgase am Gesamtoutput sind vernachlässigbar $<0.05$ t). Die Zinkverteilungen unterscheiden sich nicht stark von den Werten für 1996 (vgl. Abbildung 3.2.4).

Die mittlere Konzentration des Zinks im Siedlungsabfall beträgt $2.1 \pm 0.5 \mathrm{~g} / \mathrm{kg}$. Sie ist leicht höher als die in anderen Studien erhaltenen Werte, aber in der gleichen Grössenordnung [9.10|. Die relativ hohe Unsicherheit der Zinkkonzentration in der Schlacke $(4.9 \pm 1.9 \mathrm{~g}$ ) $\mathrm{kg})$ rührt von den zeitlichen Variationen der Konzentrationen in den Feinfraktionen (Abbildung 3.2.5) sowie von der Unsicherheit der Konzentrationsbestimmung im Grobgut und im Siebrest her (Abschnitt 2.6.4). Der relative Fehler der Zinkkonzentration im Verfestigungsprodukt ist ca. 15\%. Die mittlere Zinkkonzentration im Abwasser ist kleiner als die Einleitbedingung in ein Gewässer ( $2 \mathrm{mg} / 1, \mid 15)$, und das Abgas erfüllt die Luftrein halteverordnung $|12|$.

Diese Resultate bestätigen, dass bezüglich der Zinkflüsse Analysen von 3-Monats-Mischproben von Klärschlamm, Dickschlamm und Abwasser genügen würden (Tabelle 2.4.1). Wöchentliche Analysen können jedoch Informationen über die Prozesse liefern. Zum Beispiel kann die zeitliche Abnahme der Zinkkonzentration im Klärschlamm oder der "Ausreisser" im Abwasser (Abbildung 3.2.5) wertvolle Hinweise über die kommunale Abwasserreinigung [4]. und die Abwasser aufbereitungsprozesse in der MVA geben

\subsubsection{Prozess-Controlling}

Die Verteilung des im Siedlungsabfall und in den Schlämmen vorhandenen Zinks zwischen der Schlacke, den Rückständen der Rauchgasreinigung (RRGR), dem Abwasser und dem Abgas kann nicht direkt aus der Abbildung 3.2.6 herausgelesen werden. Aus diesem Grund wurde bei einem weiteren Schritt die Systengrenze verändert und die Reststoffverfestigung ausgeklammert. Abbildung 3.2.7 zeigt die Resultate. Der grösste Teil $(k=0.97 \pm 0.003)$ des Zinks fliesst in die Anlage via Siedlungsabfall, da die Fremdreststoffe nicht über die Verbrennungsöfen in die Anlage fliessen und deshalb nicht berücksichtigt werden. Klärschlamm und Dickschlamm liefern den Rest. Zink gelangt je zur Hälfte in die Schlacke und in die Rückstände der Rauchgasreinigung. Diese Verteilung stimmt mit den Literaturwerten gut überein $\{9,10\}$.

Eine wichtige Frage ist, ob Prozessvariationen in Transferkoeffizienten feststellbar sind. Tabelle 3.2.3 zeigt einen Vergleich der Transferkoeffizienten von Zink in der gesamten Messperiode mit zwei Teilperioden. Die unterschiedlichen Transferkoeffizienten in

\section{Zinkfluß ohne} Verfestigung (in Tonne pro Bilanzperiode Jahr: 1997

Woche: 1 bis und mit 18

Siedlungs-
abfall
$42.3 \pm 10.4 \quad$ Rostofen
$(2.05 \pm 0.51 \mathrm{~g} / \mathrm{kg})$
$(k=0.97 \pm 0.03)$
$(k=0.97 \div 0.03)$

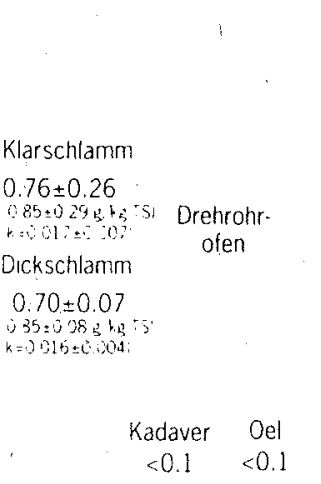

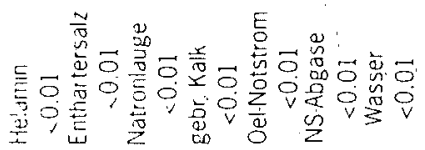

Kessel, Energieruckgewinnung, Abgasbehandlung,

Hilfsmittelzubereitung und verteilung, Aschewaschung, Abwasserremigung

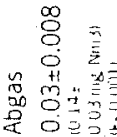

TMT 15

$<0.01$

FHM

$<0.01$

Salzsaure $<0.01$

$\begin{array}{cc}\text { Brauch- } & \text { Brauch. } \\ \text { wasser- } & \text { wasser } \\ \text { verteilung } & <0.01\end{array}$

\begin{tabular}{|c|c|c|}
\hline $\begin{array}{c}\text { Kläschlamm- } \\
\text { schlackenbad } \\
\text { Schlackenbad } \\
\text { Bunker }\end{array}$ & , : & $\begin{array}{l}\text { Reststoff. } \\
\text { vertellung }\end{array}$ \\
\hline , & & $\begin{array}{c}\because \\
y^{2}\end{array}$ \\
\hline $\begin{array}{c}\text { Schlacke } \\
22.6 \pm 8.9 \\
j=15 k_{0} 151 \\
k=1)+10 \pm 1\end{array}$ & $\begin{array}{c}\text { Abwasser } \\
0.0124 \pm 0.0171 \\
0+3 \pm 0.60 \mathrm{mlg} \cdot 11 \\
k .0001 \mathrm{i}\end{array}$ & $\begin{array}{c}\text { Verfestigungsprodukt } \\
1 \quad \begin{array}{l}33.2 \pm 5.2 \\
193 \pm 3.1 \mathrm{~kg}(\mathrm{si}) \\
k=139 \pm 13.101\end{array}\end{array}$ \\
\hline
\end{tabular}

FRST

$12.1 \pm 1.29$

Kakkydrat

$<0.01$

Zement

$0.04+0.001$

Systemgrenze den Teilperioden würden auf Veränderungen der chemischen Speziierungen des Inputs (Siedlungsabfall) und/oder auf Veränderungen der Prozessbedingungen in der Anlage hinweisen In den ersten 9 Wochen (Teilperiode 1) ist der mittlere Transferkoeffizient in die Schlacke (0.57) grösser als der mittlere Transferkoeffizient in die Rückstände der Rauchgasreinigung (0.43). In den Wochen 10 bis 18 (Teilperiode 2) ist es umgekehrt (0.46 gegenüber 0.54). Es kann jedoch nicht signifikant festgestellt werden (5\% Irrtumwahrscheinlichkeit), ob ein Unterschied in beiden Teilperioden be-

Zinkfluß ohne Verfestigung (in Tonne pro Bilanzperiode) Jahr: 1997

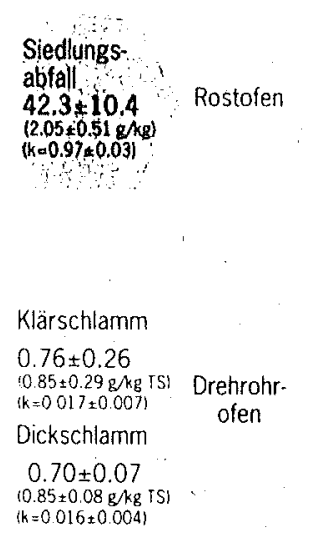

Kadaver Oel $<0.1<0.1$
Woche: 1 bis und mit 18
Aboildung 3.2.6: Zinkfluß in den ersten 18 Wochen. Flüsse innerhalb des Systems werden nicht wertproportional dargestellt. FRST: Fremdreststoff

Abbildung 3.2.7 Zinkflu $B$ in den ersten 18 Wochen ohne Berücksichtigung der Reststoftverfestigung. Flüsse innerhalb des Systems werden nicht wertproportional dargestellt.

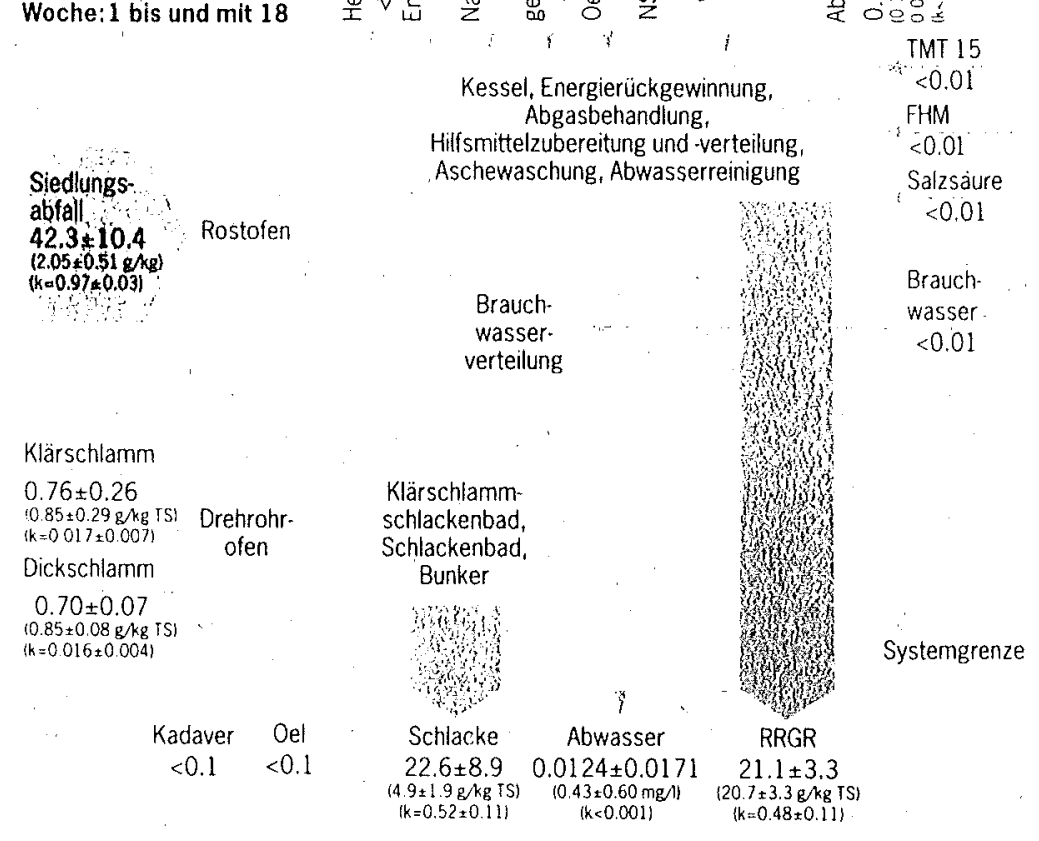




\begin{tabular}{llll}
\hline $\begin{array}{l}\text { Zeitperiode } \\
\text { Woche) }\end{array}$ & \multicolumn{2}{l}{ Transferkoeffizient von Zink } \\
& in der Schlacke & $\begin{array}{l}\text { in die Rückstände der } \\
\text { Rauchgasreinigung (RRGR) }\end{array}$ \\
\hline 1 bis 9 (Teilperiode 1) & $0.57 \pm 0.12$ & $0.43 \pm 0.12$ & $<0.001$ \\
\hline 10 bis 18 (Teilperiode 2) & $0.46 \pm 0.24$ & $0.54 \pm 0.24$ & $<0.001$ \\
\hline 1 bis 18 (Gesamtperiode) & $0.52 \pm 0.11$ & $0.48 \pm 0.111$ & $<0.001$ \\
\hline
\end{tabular}

Tabelle 3.2.3:

Transferkoeffizienten

von Zink in verschie-

denen Zeitperioden

steht, da die Anzahl Messungen klein ist (z.B. nur 2 Punkte für RRGR a der Teilperiode 2). Mit einer erhöhten Probenanzahl könnten jedoch verfahrenstechnische Variationen festgestellt werden [11].

\subsection{Der ökonomische Aufwand}

Der Zeitaufwand für die Güterbuchhaltung wird auf 40 und für die Probenahme auf 30 Arbeitsstunden pro Jahr geschätzt. Dieser Aufwand wird vom MVA-Personal aufgebracht. Die Güterbuchhaltung und die Probenahme werden jedoch mit den alltäglichen Aktivitäten wie z. B. Aufladen der Schlacke aus dem Bunker auf den Lastwagen kombiniert. Probenaufbereitung und chemische Analysen sind dagegen zusätzliche Arbeiten. Je nach Anzahl der gemessenen Elemente belaufen sich die Kosten für diese in externen Laboratorien durchgeführten Arbeiten zwischen $20^{\prime} 000$ und 30'000 SFr pro Jahr, oder maximal $0.5 \mathrm{SFr}$ pro Tonne Abfall. Eine solche Stoffbuchhaltung würde also die Gesamtkosten (Transport + Behandlung + Deponie) von 300-500 SFr pro Tonne (Schweizer Zahlen) nur im Promillbereich erhöhen,

\section{Zusammenfassende Schlussfolgerungen}

Die vorgestellte Methode erlaubt die Güter- und

Stoffflüsse in einer Müllverbrennungsanlage kontinuierlich zu erfassen.

\section{Nevauflage}

\section{Straßenreinigung und Winterdienst in der lommunallen Praxis}

\author{
RECHTSGRUNDLAGEN - ORgANISATION - AUFGABEN \\ VON Dr. MANFRED WICHMANN, Referent beim \\ Deutschen Städte- und Gemeindebund \\ 2., neubearbeitete und wesentlich erweiterte Auflage 1997, \\ 432 Seiten, $15,5 \times 23,5 \mathrm{~cm}$, fester, foltenkaschierter Einband, \\ DM 148,-/öS 1.080,-/sfr. 131,-. ISBN 3503043179
}

In dem Buch werden die Rechtsgrundlagen für Straßenreinigung und Winterdienst in Städten und Gemeinden exakt und ausführlich unter Einbeziehung einschlägiger Veröffentlichungen und vieler aktueller Grundsatzentscheidungen der Obergerichte und des Bundesgerichtshofes dargestellt. Des weiteren werden Probleme aus der Praxis geschildert, die im Rahmen der behandelten Rechtsgebiete auftreten, sowie Lösungsmöglichkeiten aufgezeigt. Durch zahlreiche Beispiele, Vertrags- und Satzungsmuster sowie Anhänge ergänzt, ist das Buch ein kompetenter Ratgeber und ein ideales Nachschlagewerk für die Kommunen sowie für den reinigungspflichtigen Bürger.

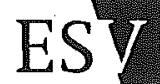

Der ökonomische Aufwand für die Bestimmung der Stoffkonzentrationen ist, gemessen an den Gesamtkosten, sehr gering $(<1 \%)$.

Die Informationen, welche aus den Stoffflüssen (ausgewählte Indikatorelemente) gewonnen werden können, erlauben eine differenzierte Beurteilung von

a) Input/Output-Bilanzen

b) Prozesstechnischen Eigenschaften

c) Wirkungen von stofflichen Massnahmen im Einzugsgebiet der Müllverbrennungsanlage.

\section{Literaturangaben}

[1] Brunner P.H \& Baccini P.:

Regional materials management and environmental protection Waste Management and Research. 10, 203-212, 1992.

[2] Baccini P., Daxbeck $\mathrm{H}_{\text {, }}$ Glenck E. \& Henseler $\mathrm{G}$.:

Metapolis - Güterumsatz und Stoffwechselprozesse in den Privathaushalten einer Stadt. Bände $34 A+B$, Nationales Forschungsprogramm Stadt und Verkehr, Zürich, 1993.

(3) Baccini P. \& Bader H.-P.:

Regionaler Stoffhaushalt, Spektrum Akademischer Verlag, Heidelberg, Berlin, Oxford, 1996

[4] Chassot G.M., Besson J-M., Candinas T., Baccini P., Henseler G. Siegrist H.:

Modifications métaboliques anthropiques, Gas. Wasser, Abwasser, 76, 409-415, 1996

[5] Baccini P., Belevi H. \& Lichtensteiger Th. Deponie in einer ökologisch orientierten Volkswirtschaft, GAIA, 1 34-49, 1992.

(6) Enquete-Komission:

Grundlagen des Stoffstromm-Managements. Die Industriegesell. schaft gestalten - Perspektiven für einen nach haltigen Umgang mit Stoff- und Materialströmen. Hrsg. von Enquete Kommission Schutz der Menschen und der Umwelt" des Deutschen Bundestages, Economica Verlag, Bonn, Deutschland, 1994.

(7) Baccini P. \& Brunner P:

Metabolism of the Anthroposphere, Springer, Berlin, 1991.

[8] Henseler G., Bader H.-P., Oehler D., Scheidegger R. \& Baccini P.: Methode und Anwendung der betrieblichen Stoffbuchthaitung, vdf Hochschulverlag an der ETH Zürich, 1995

(9) Brunner P.H.\& Moench H.:

The flux of metals through municipal solid waste incinerators. Waste Management \& Research, Vol. 4, p. 105-119, 1986

[10] Belevi H::

Was können Stoffflussstudien bei der Bewertung der thermischen Abfallbehandlung leisten?, VDi-Berichte Nr. 1033, 261-276, 1993.

[11] Belevi $\mathbf{H}$

Trace elements provide insight into solid waste combustion processes", EAWAG News 40E, p. 19--22, 1996.

12|Schweizerischer Bundesrat: Luftreinhaite-Verordnung [LRV], 1992.

[13| Wurzschmitt B.:

Ein neues Schnellverfahren mit Alkaliperoxid in einer Universalbombe für Mikro-, Halbmikro- und Makroeinwaagen, Microchimica Acta Vol. 36/37, p. 369. 1951

[14] Sachs L.:

Angewandte Statistik, Springer Verlag, Berlin, 1992

[15]Schweizerischer Bundesrat;

Verordnung über Abwassereinleitungen, 1988.

[16] BUWAL:

Empfehlungen über die Emissionsmessungen von Luftfremdstoffen bei stationären Anlagen, Bern, Schweiz, 1987.

(17) Belevi $\mathrm{H}$ :

Einfluss von Inputqualität und Verfahrenstechnik auf die Qualität der Produkte in Müllverbrennungsanlagen, in Vorbereitung.

[18] Belevi H., Stämpfli D. M. \& Baccini P.

Chemical behavior of municipal solid waste incinerator bottom ash in monofills, Waste Management \& Research, Vol. 10, 153-167, 1992.

\section{Anschrift der Autoren:}

Dr. Hasan Belevi, Madeleine Langmeier, Hermann Moench, Prof. Dr. Peter Baccini

EAWAG. CH.8600 Dübendorf Schweiz

Tel.: 004118235514

e-mail, belevi@eawag.ch

Yvonne Turban, Thomas Müller

Kehrichtverbrennungsanlage Oftringen

Altestrasse $40, \mathrm{CH}-4665$ Oftringen, Schweiz 